(Aus dem physiologischen Institut der Universität Strassburg i. E.)

\title{
Untersuchungen \\ über Tonus, Hemmung und Erregbarkeit.
}

Von

G. A. Jäderholm (Stockholm).

(Mit 2 Textfiguren und Tafel II--IV.)

Inhaltsübersicht:

A. Einleitung. . . . . . . . . . . . . . . 249

B. Tonus. . . . . . . . . . . . . . . . . 250

I. Methodik der Tonusuntersuchungen . . . . . . . . . 250

II. Tonische Kontraktionen. . . . . . . . . . . . . . . . . . . . 256

a) Regel der doppelten Möglichkeit . . . . . . . . . . 256

b) Beweis für die nervöse Bedingtheit der tonischen Kontraktion 260

c) Einfluss der Dehnung auf das Zustandekommen tonischer Kontraktionen . . . . . . . . . . 261

d) Literatur . . . . . . . . . . . . . . . . . 262

C. Die Beeinflussung der tonischen Zustände und der direkten Erregbarkeit des Nerven durch dynamogene und inhibitorische Reize . . . 265

I. Methodisches ................ 265

II. Schwellenschwankung und Schwellenwanderung. . . . . . . 266

III. Mittel, Hemmungen hervorzurufen . . . . . . . . . . . . . . . 269

IV. a) Hemmung und Förderung tonischer Kontraktionen . . . . . 272

b) Literatur über Förderung und Hemmung tonischer Kontraktionen 277

V. Beeinflussung der Erregbarkeit des peripheren Nerven durch reflektorische, besonders hemmende Reize . . . . . . . 279

a) Reine Erregbarkeitsänderungen . . . . . . . . . . 279

b) Komplikation der Erregbarkeitsänderung des Muskelnerven mit tonischen Phänomenen . . . . . . . . . . 282

c) Weitere Komplikationen . . . . . . . . . . . . . 285

d) Nachwirkungen . . . . . . . . . . . . . . . . 288

VI. Literatur über die Beeinflussung der Erregbarkeit des peripheren

Nerven durch refiektorische, besonders hemmende Reize . . . 290

D. Theoretisches; Zusammenfassung . . . . . . . . . . . 297 


\section{A. Einleitung.}

Im Jahre 1898 publizierte J. Starke ${ }^{1}$ ) eine vorläufige Mitteilung „über den Finfluss des zentralen Nervensystems auf die Erregbarkeit der motorischen Nerven". Eine versprochene, ausführlichere Darstellung der Methoden und der betreffenden Phänomene ist ausgeblieben, und dem genannten kurzen Referat müssen darum die betreffenden Angaben entnommen werden:

Chemische Reizung gewisser Teile des zentralen Nervensystems beim Frosch ruft Senkung der Erregbarkeit der peripheren Nerven hervor. Die Erregbarkeitsänderung wurde an der Zuckungskurve gezeigt, welche durch regelmässige Reizung des einen Ischiadicus mit Öffnungsinduktionsschlägen hervorgerufen wurde. Bei Reizung der zentralen Wurzelstumpfe des anderen Ischiadicus erhielt der Verfasser nie Senkung der Erregbarkeit, dagegen wiederholte Male eine Erhöhung.

Zwei Jahre später veröffentlichte M. V erw or ${ }^{2}$ ) eine Kontrolluntersuchung, durch welche er durchgängig zu negativen Resultaten gelangt war. Wenn das Gehirn nach den Angaben Starke's unter allen denkbaren Kautelen chemisch gereizt wurde, so hatte dies nicht den geringsten Einfluss auf die Höhe der Kontraktionskurven, die vom Gastrocnemius durch Metronomreizung vom Nerven aus zu erhalten waren. Die Kontraktionskurven blieben sich auch gleich nach Abtrennung des Grosshirns, bei Reizung, Durchschneidung oder Zerstörung tieferliegender Teile des zentralen Nervensystems und bei Reizung des Nervus Ischiadicus der entgegengesetzten Seite auch dann, wenn deutliche Hemmungseffekte eintraten.

Andrerseits lag eine Reihe italienischer Untersuchungen (ausgeführt von Belmondo und $O d d^{3}$ ), $O d d^{4}$ ), später in $O d d i ' s$

1) J. Starke, Über den Einfluss des Zentralnervensystems auf die Erregbarkeit der motorischen Nerven. Physiol. Zentralblatt Bd. 12 Nr. 18 S. 596 bis 599.1898.

2) M. Verworn, Zur Physiologie der nervösen Hemmungserscheinungen. Arch. f. Physiologie. Supplem. 1900 S. 105-124.

3) E. Belmondo und R. Oddi, Intorno all' influenza delle radici spinali posteriori sull eccitabilità delle anteriori. Rivista sperim. vol. 16 (Sep.).

4) R. Oddi, Il cervello ed il midollo spinale come centri di inibizione. Rend. della R. Acad. dei Lincei 4 Serie 5 a, Fasc. $6^{\circ}$ (Sep.). - R. Oddi, L'inibizione dal punto di vista fisio-patologico, psicologico e sociale. Torino 1898. 
Laboratorium von Manelli ${ }^{1}$ ) und Polimanti ${ }^{2}$ ) u. a.) an Warmblütern vor, die nach den Darstellungen der Autoren unter genügenden Kautelen ausgeführt worden waren, und welche zeigten, dass der Reizzustand der hinteren-Wurzeln einen deutlichen Einfluss hat auf die Erregbarkeit der vorderen, und zwar so, dass Durchschneidung der Wurzeln Erregbarkeitssenkung, Reizung der zentralen Wurzelstücke Erhöhung der Erregbarkeit oder, bei stärkerer Reizung, Herabsetzung auf der anderen Seite hervorrufen.

Die Frage, ob die E'rregbarkeit der motorischen Nerven durch reflektorische Einflüsse von seiten des zentralen Nervensystems, besonders in depressiver Weise, verändert werden kann, harrte also der definitiven Entscheidung. Einige orientierende Versuche, welche Professor Bethe im hiesigen Institut anstellte, sprachen zugunsten der Auffassung der italienischen Forscher. Nach Verbesserung der Methodik und genauerer Prüfung aller Kautelen ist es mir gelungen folgendes mit Sicherheit festzustellen: Bei Reizung des Ischiadicus mit gleichgerichteten und gleichstarken Induktionsschlägen von genügender Reizstärke variiert die Zuckungshöhe des Gastrocnẹmius gar nicht; werden jetzt hemmende Reize dem zentralen Nervensystem zugeführt, so tritt in sehr vielen Fällen eine Erniedrigung der Zuckungshöhe, resp. vollkommener Ausfall einiger Zuckungen ein. Während des Verlaufes dieser Untersuchung wurde es mir aber sehr bald klar, dass gewisse orientierende Untersuchungen über die Rückenmarksfunktionen im allgemeinen, besonders über den "Tonus", für das Verständnis dieses Phänomens unbedingt nötig waren. Ich teile jetzt. zuerst die letztgenannten Befunde mit; im späteren Teil meiner Darstellung werde ich die Hemmungsphänomene demonstrieren.

\section{B. Tonus.}

\section{Methodik der Tonusuntersuchungen.}

Untersuchungen aber die Funktionen des zentralen Nervensystems haben ja bekanntlich immer mit Schwierigkeiten zu kämpfen, die beim Experimentieren mit anderen Organen wohl auch zum Teil

1) Manelli , Sopra alcuni fatti di inibizione riflessa etc. Riv. sperim. vol. 22. Fasc. $1^{\circ}$ (Sep.). 1896.

2) 0. Polimanti, Influenza che le radici posteriori esercitano sulla eccitabilità delle anteriori. Bull. Acad. med. di Roma vol. 22 (Sep.). 1896. 
vorkommen, die aber beim zentralen Nervensystem ungewöhnliche Grössen erreichen. Wenn man das Zentralnervensystem aus dem Körper der Tiere entfernt, und dessen Funktionen ausserhalb des Körpers untersuchen will, so entstehen eine Menge von Wundflächen, die Zirkulation hört auf, und das Organ stirbt auch unter Innehaltung grösster Vorsichtsmassregeln schnell ab. Was für einen Wert die Resultate solcher Untersuchungen für unsere Erkenntnis der normalen Vorgänge haben, leuchtet ja von selbst ein. Aber wenn man andrerseits das Organ im Tierkörper lässt, treten auch erhebliche Schwierigkeiten auf. In jedem Augenblick treten Reize durch die verschiedensten afferenten Bahnen ins Zentralorgan ein, lösen motorische Impulse aus, und durch die so hervorgerufenen Bewegungen entstehen wieder neue Reize. In keinem Augenblick herrscht dynamisches Gleichgewicht im Zentralorgan. Es sind die Tiere in dieser Beziebung viel empfindlicher, als man gewöhnlich glaubt. Eine Fesselung ist oft genügend, um eine relativ starke Hemmung hervorzurufen. Es gehören zwar diese Hemmungen nicht in die Kategorie von Hemmungen, durch welche die Tiere reflexlos werden, aber die Antwort auf die Reize kommt beträchtlich später und träger, wie man schon an den Kornealreflexen eines aufgebundenen Frosches beobachten kann ${ }^{1}$ ). - Bei Untersuchungen über die Funktionen des Rückenmarks werden ja von alters her die höheren Zentren durch eine quere Durchtrennung unterhalb der Medulla oblongata aus dem Spiel gesetzt. Eine derartige Operationsweise,

1) Es mag hier gleich ein Autor genannt werden, der diese Tatsachen bereits beobachtet hat, und bei dem man die Literatur für das betreffende Gebiet kritisch erörtert findet. Schrader (Über das Hemmungszentrum des Froschherzens und sein Verhalten in Hypnose und Shock. Diss. Strassburg 1886) untersuchte den Einfluss von gewissen Allgemeinzuständen des Zentralnervensystems auf die Herzbewegung und stellte dabei folgende zwei Zustandstypen des Nervensystemıs auf. Unter Shock verstand er - des prägnanten Ausdruckes wegen (unter Verzicht auf jede Beziehung zu dem Shock der Chirurgen) eine plötzliche, wie mit einem Schlage eintretende Zustandsänderung des Zentralnervensystems, welche hervorgerufen wird durch direkte mechanische Erschütterung der Zerebrospinalachse oder durch einen starken peripheren Nervenreiz. Als Hy pn os e dagegen bezeichnet er denjenigen Zustand, welcher sich bei einem Frosch einstellt, wenn man ihn schnell in eine ungewohnte Körperhaltung versetzt und ihn in derselben, falls noch Widerstand geleistet wird, so fixiert, dass kein direkter Druck auf das Tier ausgeübt wird, das aber jede Bewegung alsbald auf einen festen Gegenhalt stösst. 
ebenso wie die Anwendung von Strychnin, schien mir für meine Zwecke, aus leicht ersichtlichen Gründen, unzweckmässig.

Ich habe daher, auf Rat von Prof. Bethe, eine Versuchsmethode gewählt, die in möglichst hohem Grade Wundflächen, Zirkulationsaufhebungen und Reize von der Peripherie bei ganz erhaltenem Zentralnervensystem vermeidet. Es kam ja vor allem darauf an, das Tier möglichst reizlos zu fesseln und keine Gelegenheit zur Entstehung neuer Reize durch den Widerstand bei den Fluchtversuchen zu geben: dem Tiere - bei allẹn diesen Experimenten dem Frosche - wurden wenigstens vier oder fünf Tage vor dem Experiment die sensiblen Rückenmarkswurzeln des linken Ischiadicus durchschnitten und die Wunde sorgfältig zugenäht. Selbstverständlich wurden nur solche Tiere benützt, bei welchen die Sensibilität vollkommen verschwunden war, bei erhaltener Beweglichkeit des Beines. Das Tier wurde zum Versuch auf eine Quecksilberoberfläche $(18,8 \times 21,5 \mathrm{~cm})$ gesetzt und mit zwei Klemmen in der Weise befestigt, dass die eine den blossgelegten Ferour des asensiblen Beines, die andere den Unterschenkel nahe am Fussgelenk hieit (siehe Fig. 1 S. 253). Die Klemmen waren mit starken Kreuzköpfen an einem Stativ befestigt, dessen Fussplatte aus einem grossen, $5,8 \mathrm{~kg}$ schweren Stück Blei bestand. Wenn das Quecksilberbad genügend tief ist $(1,5-2 \mathrm{~cm})$, findet das Tier bei seinen Bewegungen keinen festen Punkt, um auf die Versuchsanordnung bei seinen Fluchtversuchen mechanisch einzuwirken. Es versteht sich übrigens von selbst, dass dieser Vorrichtung auch sonst eine solche Festigkeit gegeben wurde, dass jede Fehlerquelle aus Verschiebungen usw. ausgeschlossen war.

So gefesselt, werden die Fesselungsreize gegenüber anderen Methoden erheblich reduziert; in den ersten Augenblicken nach der Fesselung treten natürlicherweise heftige und starke Bewegungen auf, aber beim Tier mit durchschnittenen hinteren Wurzeln hören sie viel früher auf als bei normalen Tieren. Das Versuchstier liegt dann mit dem anderen Bein in Hockstellung und ohne Zeichen von Hemmung ruhig auf dem Quecksilber. Die Kornealreflexe erfolgen ebenso schnell wie beim ungefesselten Tier. - Für gewisse von den hier mitgeteilten Versuchen genügte auch eine einfachere und weniger zeitraubende Methode, um gute Resultate zu bekommen. [Bei den betreffenden Versuchen wird unten mitgeteilt, dass sie so ausgefübrt wurden]: Das Versuchstier wurde dabei auf eine Korkplatte mit 
schräggestellten Nadeln befestigt: Besonders brauchbar war diese Methode bei Tieren, die schon mehrere Tage lang stark abgekühlt waren; die Trägheit der Bewegungen ist bei diesen Tieren ausserordentlich gross, und es scheint ihnen relativ gleichgultig, ob ihre Beine nach hinten ausgestreckt sind, wenn sie nur ruhig liegen dürfen. - Die Achillessehne wird vor der Fesselung möglichst lang abgeschnitten, ein Haken wird in dieselbe eingehakt, und von diesem geht ein ca. $30 \mathrm{~cm}$ langer und sehr dünner Faden durch eine Spalte in der Wand der Quecksilberwanne zu einem borizontal auf einer

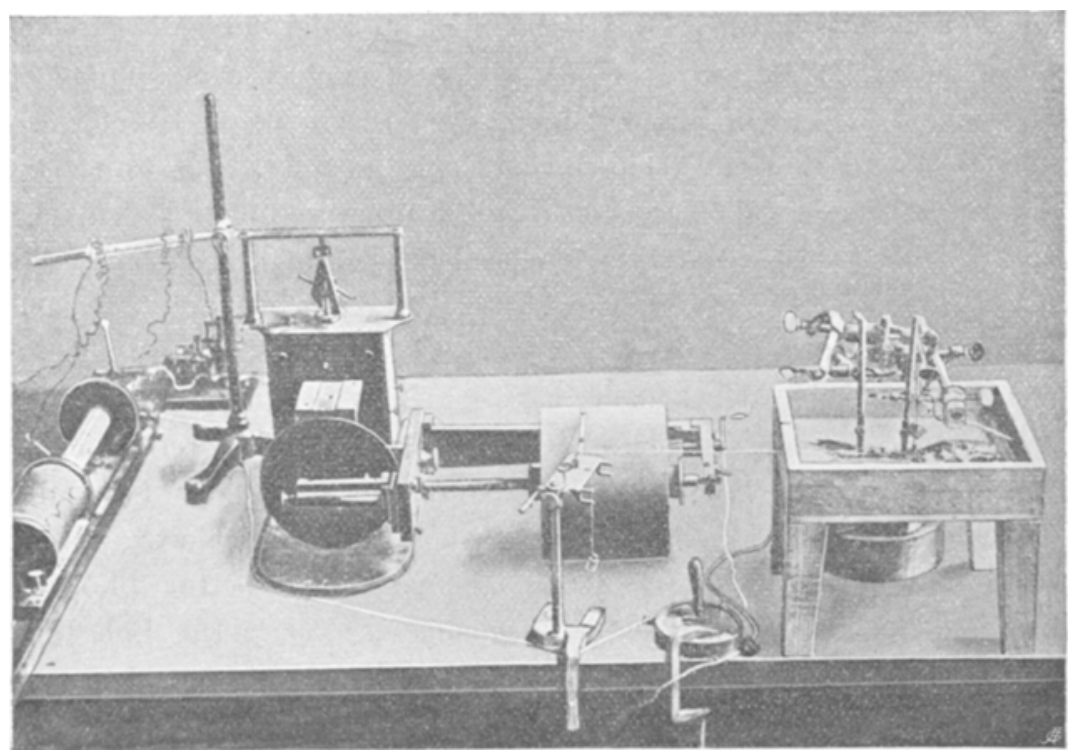

Fig. 1.

Kymographiontrommel schreibenden Hebel obne Anschlag [M a r e y $\left.{ }^{1}\right)$ ]. Um keine Fehlerquellen durch die Kontraktionen der Oberschenkelmuskulatur zu bekommen, müssen die Haut und die Faszien zwischen Ober- und Unterschenkel durchschnitten werden. Die in einigen Fällen durchgeführte Durchschneidung aller Sehnen der Oberschenkelinuskeln an Kniegelenk ist nicht unbedingt erforderlich ${ }^{2}$ ), und wegen

1) Marey, La Methode graphique p. 193. Paris 1878.

2) Versuche mit durchschnittenen Sehnen der Oberschenkelmuskulatur führten zu genau denselben Resultaten wie die anderen Versuche. 
der leicht entstehenden Blutung schwierig. Blutungen sind natürlich peinlichst zu vermeiden, um den Muskel nicht durch Zirkulationsstörungen in seiner Tätigkeit zu beeinträchtigen. (Die Haut über dem Gastrocnemius spaltet man am besten in der Längsrichtung des Muskels.) Bei der Durchschneidung der Achillessehne müssen auch nach Möglichkeit Dehnungen des Gastrocnemius vermieden werden, wenigstens wenn man normale tonische Kontraktionen.erzielen will.

In der Mehrzahl der hier mitgeteilten Versuche war es wichtig, die Reibung des Hebels auf der Trommel möglichst zu beseitigen. Es gelang dies auch durch eine einfache, von J. R. Ew ald (1879) ${ }^{1}$ ) angegebene Vorrichtung: Der Hebel wird in jeder Sekunde zweimal durch einen Faden, der zu einem Uhrwerk geht, von der Trommel abgehoben. Der sehr dünne Faden war $175 \mathrm{~cm}$ lang, konnte also keinen nennenswerten Widerstand gegen horizontale Exkursionen der schreibenden Spitze leisten. Die Kurven bestehen daher nur aus Strichen; weil jeder Strich eine halbe Sekunde bedeutet, hat man so auch die Zeitschreibung. Bei der Untersuchung der lang ausgezogenen tonischen Kontraktionen riefen die Unterbrechungen der Kurven keine Übelstände hervor. [Die Strichkurven wurden sehr dünn gezogen, so dass sie sich nicht ohne weiteres photographisch kopieren liessen. Ich habe darum unter der Lupe die einzelnen Striche mit einer sehr spitzen Nadel retuschiert.] - Die Länge des Hebels vom Drehpunkt bis zur Spitze betrug $14,5 \mathrm{~cm}$, bis zum Angriffspunkt des Gastrocnemius $2,8 \mathrm{~cm}$. Die Belastung wirkte auf die Achse (Diameter $0,4 \mathrm{~cm}$ ) mit Überführung über eine zweite Rolle. Normale Belastung für die Tonuskurven war $20 \mathrm{~g}$; d. h. für den Muskel 1,48 g. Für die speziellen Belastungsexperimente benutzte ich 30,50 oder $100 \mathrm{~g}$; auf dem Muskel hat dabei also in der Wirklichkeit eine Belastung von 2,1, 3,57 oder $7,14 \mathrm{~g}$ gewirkt.

$\mathrm{Zu}$ den Versuchen wurden Winterfrösche und Sommerfrösche, ebenso wie mehrere Tage abgekühlte oder ebenso lang warmgehaltene (plus $28^{\circ}$ C.) Tiere benützt, und zwar. von beiden Arten, R. temporaria und esculenta. Für Tonusversuche dürften schon die Temporarien etwas mehr geeignet sein als die Esculenten. Doch

1) J. R. Ewald, Eine neue Methode, den Druck in der Lunge zu messen. Pflüger's Arch. Bd. 20 S. 262-264. 1879. 
war der Unterschied kleiner, als nach den Versuchen Verworns $\left.(1897)^{1}\right) \mathrm{zu}$ erwarten wäre.

Elektrische, mechanische, chemische, photische und akustische Reize wurden angewandt. Zum photischen Reizen wurde über die Quecksilberwanne ein innen geschwärzter Holzkasten gestülpt, der auf einer Seite eine Spalte für den Faden zum Gastrocnemius, auf der entgegengesetzten ein Fenster hatte, das vermittelst einer Kartonplatte geschlossen und geöffnet werden konnte. Akustische Reize fand ich unwirksam, so dass die Art ihrer Herstellung übergangen werden kann. Die elektrischen und mechanischen Reize wurden auf die Rücken-, Bauch- oder Beinhaut der rechten Seite des Tieres appliziert; es sei daran erinnert, dass das Tier immer nur mit dem linken Bein gefesselt war. Mechanische Reize waren Kneifen der Haut mit einer starken Pinzette, Massage, d. h. nicht zu sanftes Streicheln der Rückenhaut, und Betupfen der Corneae. Elektrisch gereizt wurde auch der Ischiadicus der rechten Seite, sowohl mit Einzelschlägen als auch faradisch. Zur Zuführung wurden versenkbare Elektroden benutzt; zur Reizung diente ein kleiner Induktionsapparat (Rollenlänge $6 \mathrm{~cm}$ ), in Verbindung mit einem kleinem Grenetelement. Mit denselben Nadelelektroden, die (neben Lamettafäden) zur Zuführung der elektrischen Hautreizung gebraucht wurden, spüre ich auf der Fingerhaut, auch bei grosser Unterbrechungsfrequenz, die Ströme erst bei einem Rollenabstand von $4,1 \mathrm{~cm}$. Es wird daher nicht wundernehmen, wenn ich zur Hervorrufung mancher Effekte Rollenabstände bis zu $0 \mathrm{~cm}$ angewandt habe. - Die chemischen Reize endlich wurden gewöhnlich durch eine Injektion von 10\% iger Kochsalzlösung in den Rückenlymphsack zugeführt. Die Lösung wurde mit einer Pravaz'schen Spritze eingeführt; die Menge betrug in der Regel $1 / 2-1 \mathrm{ccm}$; mit weniger als $0,3 \mathrm{~cm}$ waren keine Resultate zu erzielen. Um starke Wirkungen zu erzielen, empfiehlt es sich, die Rückenhaut etwas zu massieren (s. S. 270).

Sehr wichtig ist es, dass man das Tier nicht mit zu starken und zu vielen Reizen malträtiert; es ändert sich dann, nach meinen Erfahrungen, sehr leicht die Reaktionsweise des Zentrums. Auch darf man das Tier nicht im direkten Sonnenlicht sitzen lassen. Dagegen erwies es sich bei photischer Reizung vorteilhaft, die Sonne direkt in den Kasten hineinstrahlen zu lassen.

1) M. Verworn, Tonische Reflexe. Pflüger's Arch. Bd. 65 S. 63-80, S. 64.1897. 
Noch eine Komplikation der Methodik ist hier anzuführen. Bei gewissen Phänomenen wurde nämlich der linke Ischiadicus auch freigelegt und regelmässig, etwa einmal in der Sekunde, mit Einzelschlägen gereizt. Genauere Angaben über die benützte Methodik finden sich unter Methodisches zu den Hemmungsuntersuchungen. -

Bei den Versuchen mit Kälte- resp. Wärmetieren wurden besondere Vorrichtungen getroffen, um zu verhüten, dass die Tiere im Verlaufe des Experiments ihre Temperatur erheblich veränderten. Die Kälteversuche wurden im Winter in einem Zimmer ausgeführt, das die Aussentemperatur hatte, und wurden nur an Tagen gemacht, wo diese wenigstens bis auf plus $5^{0}$ C. gesunken war (November und Dezember 1905; Januar 1906). - Um die Wärmetiere während der Dauer des Versuches auf konstanter Temperatur zu erhalten, wurde folgende Einrichtung benützt: Eine Zinkwanne $(4 \times 12,5 \times 18 \mathrm{~cm})$ war durch eine parallel zum Boden fest angebrachte Korkplatte in eine obere und eine untere Abteilung geteilt. Auf der Korkplatte wurde der Frosch mit Nadeln befestigt; die untere Abteilung der Wanne wurde mit Wasser gefüllt, welches durch einen kleinen Bunsen-Brenner von untenher erwärmt wurde. In den oberen Raum war ein $4 \mathrm{~cm}$ hoher, unten offener Kasten eingepasst, dessen Rück- und eine Seitenwand aus Holz bestanden; in ersterer waren Löcher für Elektroden und Thermometer, in letzterer ein Schlitz für den Übertragungsfaden angebracht. Die übrigen Wände bestanden aus Glas und gestatteten die Beobachtung des Tieres. Nach oben war der Kasten mit einer gespannten Kantschuckmembran verschlossen, welche Massage gestattete, ohne den Deckel abzuheben.

Zuletzt sei noch erwähnt, dass bei der Mehrzahl dieser Untersuchungen die betreffenden Phänomene direkt beim Tier beobachtet wurden; in solchen Fällen besorgte ein Assistent die Überwachung der Apparate, und ich beschränkte meine Beobachtungen auf das Tier.

\section{Tonische Kontraktionen.}

a) Die Regel derdoppelten Möglichkeit.

Wird dem Zentralnervensystem eine Erregung zugeführt, so erfolgt, falls ein Effekt eintritt, entweder eine schnelle Reflexzuckung von grosser Hubhöbe oder eine lang ausgezogene tonische Kontraktion mit geringer Hubhöhe. 
Bei normalen Verhältnissen kann man, ohne dass es bis jetzt möglich gewesen wäre, die Ursachen der Verschiedenheit festzustellen, beide Kontraktionsformen dicht nebeneinander finden.

Kurve 3 (Taf. II.) (11. Mai 1905). Temporaria. In einzelnen Fällen wurden vergleichsweise auch Tiere ohne durchschnittene sensible Wurzeln benützt; Kurve 3 stammt von einem solchen Tier. $-K d(1-3)$ bedeutet Kneifen der Fusshaut des linken, gefesselten Beines; dabei entsteht eine tonische Kontraktion; das Loslassen $(L)$ ruft eine Zuckung hervor. Bei $K d(4)$ auf denselben Reiz eine schnelle Zuckung.

Oft reagieren vollkommen normale Tiere während einer kurzen Zeit vorwiegend tonisch, dann vorwiegend schnell, dann wieder tonisch. Das Spiel kommt oft mehrmals im Verlauf eines Experimentes vor.

Warum ein Versuchstier das eine Mal einen Reiz mit einer tonischen Kontraktion, das andere Mal mit einer schnellen Zuckung beantwortet, scheint von mehreren Faktoren bestimmt zu werden, die sich wohl nur zum Teil angeben lassen. Zum Teil wird die Antwort durch die Art des zugeführten Reizes bestimmt; es scheinen die lang ausgezogenen Kontraktionen leichter von fortgesetzten als von kurzdauernden Reizen ausgelöst zu werden. Das plötzliche Eintreten des Reizes wirkt im allgemeinen auf beide Kontraktionsarten etwas vergrössernd; doch folgt auf einen solchen Reiz, wie es scheint, leichter eine schnelle als eine tonische Kontraktion. - Wenn das Versuchstier zuerst mehrere Minuten ruhig gewesen ist und nachher eine nicht zu kurze Periode von starker Unrube gehabt hat, so ist die Reaktionsweise nicht selten verändert, und zwar oft so, dass nach einem tonischen Stadium vor der Unruhe eine Periode von Tendenz zu schnellen Zuckungen eintritt, und umgekehrt. - Das Vorwiegen der tonischen Reaktion tritt auch dann ein, wie schon oben angedeutet wurde, wenn das Tier schon mehrmals stark reagiert hat, auch im Äusseren Zeichen von Ermüdung zeigt und nicht zu kleine Reizmengen während des Verlaufes des Versuches dem Zentralorgan zugefübrt worden sind.

Photische Reize haben dann und wann Andeutungen zu tonischen Kontraktionen gegeben; ganz deutliche tonische Kontraktionen habe ich z. B. durch plötzliche Beschattung hervorrufen können.

Die tonischen Kontraktionen, die man von wirksamen Reizen bekommen kann, gehören zwei verschiedenen Typen an, die aber 
mit vielen $Z$ wischenstufen ineinander übergehen können. Beigefügtes Schema zeigt die extremen Formen.

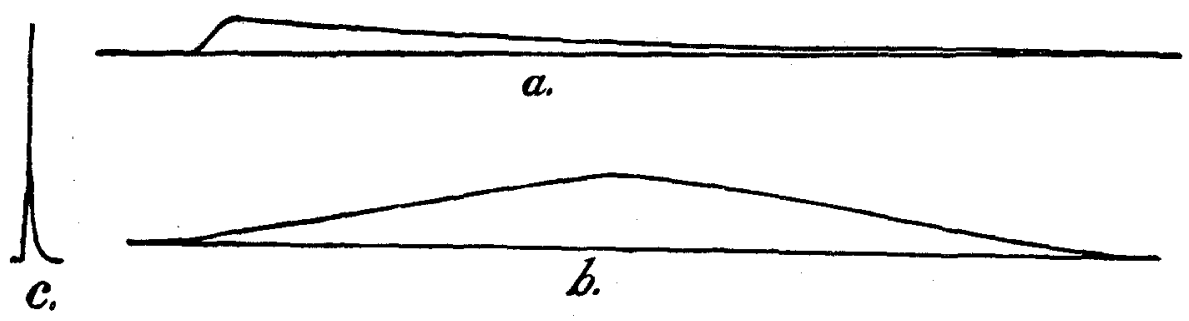

Fig. 2.

Bei Typus I (Schema $a$ ) steigt die Kurve schnell an und fällt sehr langsam ab. Bei Typus II (Schema b) ist der Anstieg nicht so schnell, dagegen ist der Abfall meistens schneller wie bei Typus I. Beide erreichen jedoch n ie die Höhe einer reflektorischen Zuckung (refiektorische Zuckung Schema c). - Die Kurven des Typus II steigen in den meisten Fällen höher wie die des Typus I.

Man bekommt den Typus I durch mechanische Reizung der Haut, z. B. durch Kneifen. Besonders leicht wurden diese tonischen Kontraktionen erhalten, wenn bei einem Tiere mit intakten hinteren Wurzeln das linke, gefesselte Bein gekniffen wurde. Es geben die Kurven 2 und 3 Beispiele.

Kurve 2 (Taf. II.) (15. Mai 1905). Esculenta. Ke Kneifen der Haut des rechten, ungefesselten Beines mit einer starken Pinzette; sensible Wurzeln links durchschnitten ( $K e 1$ tonische Kontraktion, $K e 2$ schnelle).

Kurve 3 (Taf. II.) (11. Mai 1905). Temporaria mit intakten sensiblen Wurzeln. $K p$ Kneifen der Fusshaut des linken, gefesselten Beines.

Von grossem Interesse dürften besonders diejenigen Kontraktionen dieses Typus sein, die gleichzeitig mit dem Kornealreflex auftreten. Es werden am besten gleichzeitig beide Corneae mit zwei Fingern derselben Hand plötzlich berührt.

Kurre 4 (Taf. II.) (11. Mai 1905). Esculenta. Sensible Wurzeln durchschnitten. $C 1, C 2, C 5$ Berührung der beiden Corneae, und dadurch ausgelöste tonische Kontraktionen (s. auch unten S. 259).

Elektrische Reizung der Haut mit Einzelschlägen (auch wenn sie sehr stark waren) gab nur zweifelhafte Resultate, und gewöhnlicherweise nur schnelle Zuckungen. Faradische Reize wurden dagegen oft mit Kontraktionen von dem Typus I beantwortet (Kurve $2 E e$ ). - Kleine Unterschiede existieren wohl auch zwischen den in diesen verschiedenen Weisen hervorgerufenen Kontraktionen des Typus. I. So scheinen die Kornealreizkurven immer am langsamsten abzusinken. Etwas kürzer sind gewöhnlich die durch 
mechanische Reizung und am kürzesten die durch faradische Reizung der Bein- oder Körperhaut hervorgerufenen Kurven (Kurve 2, 3 und 4).

Die chemische Reizung durch Injektion von 10\% iger Kochsalzlösung in den Rückenlymphsack gibt von allen bis jetzt gefundeuen Mitteln die längste und stärkste Tonuskontraktion. Für die Entwicklung der vollen Reizenergie empfiehlt es sich, die Haut etwas zu massieren. Die Kurve, die man dabei erhält, sowie die viel niedrigere Kurve, die man bei Massage der Rückenhaut (ohne Kochsalz) bekommt, gehören dem zweiten Typus an.

Kurve 5 (Taf. II.) (18. Mai 1905). Temporaria. $M$ Massage der Rückenhaut, bei wenig ausgeprägter Tonustendenz.

Kurve 6 (Taf. II.) (7. Mai 1905). Esculenta. P $i$ n $c$ die Rückenhaut wird mit einer Pinzette gefasst. $N$ die Nadel der Pravaz'schen Spritze wird durch die Haut eingeführt. $\mathrm{NaCl} 0,5 \mathrm{ccm} \mathrm{NaCl}(10 \%)$ wird in den Rückenlymphsack eingespritzt. Gleich danach wird die Haut losgelassen. Von $H$ ab sind deutliche Zeichen von Hemmung vorhanden (sehr langsame Kornealreflexe); sie klingt in der Gegend von $S p$ ("spontane" Zuckung) ab. (Bei diesem Versuch wurde nicht massiert!) Vgl, auch Kurve 8 (Beschreibung auf S. 261).

Ganz gewaltig, aber sehr langsam steigt auch die Kurve vom Normalniveau aufwärts, obne wieder zu sinken, - wenn im Laufe des Experiments diejenigen äusseren Symptome eintreten, die von Verworn (1897) bei Fröschen nach Reibung der Seitenhaut gefunden worden sind.

Wenn ein Versuchstier ein paarmal tonisch auf einen Reiz reagiert hat, sinkt oft während der nächsten Minuten die tonische Tendenz; dieselben Reize lösen keine sichtbare Wirkung mehr aus. Dagegen kommt es nicht selten vor, dass, wenn das Tier mehrere Minuten in Ruhe gewesen ist, der erste Reiz nicht sichtbar beantwortet wird, ein zweiter Reiz aber, der einige Augenblicke nachher kommt, sich wirksam erweist. Man darf wohl das letzte Verhältnis zugunsten einer Summation deuten; etwas Ähnliches kommt auch bei Massagekurven nicht selten vor: Erst wenn die Massage mehrere Sekunden gedauert hat, fängt die Kurve zu steigen an.

Kurve 4 (Taf. II.) (11. Mai 1905). Kornealkontraktionen. $O 1$ wirksam, $C 2$ weniger wirksam, $C 3$ nicht mehr wirksam. Zwischen $C 3$ und $C 4$ liegen etwa 9 Minuten von anpähernd vollständiger Ruhe. $C 4$ unwirksam, $C 5$ wirksam.

Kurve 5 (Taf. II.) (28. Mai 1905). Die Massagekurve steigt erst mehrere Sekunden nach Beginn der Massage vom Normaìniveau aufwärts. 
Wenn der Ischiadicus links (wie oben angedeutet, unter Methodik) gleichzeitig, etwa einmal in der Sekunde gereizt wird, so verbält sich die Zuckungskurve, von gewissen Abweichungen abgesehen, die später näher besprochen werden sollen, folgendermassen: Die Fusspunktslinie der Zuckungen steigt in die Höhe und zeigt in ihrem Verlauf dasselbe Bild wie die tonische Kontraktion.

(8. Kurve 22 Taf. III.)

Die genannten beiden Reaktionsarten, die schnelle und die tonische, kann man nach dem oben Gesagten beide nebeneinander finden; dass man bei gewöhnlicher Laboratoriumstemperatur entweder nur schnelle oder nur tonische Kontraktionen finden könnte, ist nach meinen Erfahrungen nicht zu erwarten.

Wenn man aber die Temperatur der Versuchstiere variiert, also mit erheblich abgekühlten oder erwärmten Tieren arbeitet, so zeigt es sich, dass man die beiden Reaktionsarten in ihren relativen Proportionen des Auftretens zu beeinflussen imstande ist, und.zwar ziemlich stark. Bei Fröschen, die vor dem Versuch wenigstens einige Tage auf einer Temperatur von plus 0 bis plus $5^{\circ} \mathrm{C}$. gehalten worden sind, und die während des Verlaufes des Versuchs auf relativ niedriger Temperatur erhalten werden, tritt die tonische Reaktion entschieden häufiger ein als unter normalen Umständen, wenn auch manchmal ëine schnelle Zuckung zum Vorschein kommt. Werden die Versuchstiere dagegen mehrere Tage bei einer Temperatur von plus $25-28^{\circ}$ C. gehalten, so verschwindet die tonische Reaktionsweise fast vollständig, und die Tiere reagieren fast ausschliesslich mit bald endigenden Gruppen von äusserst schnellen und energischen Zuckungen.

b) Beweis für die nervöse Bedingtheit der tonischen Kontraktion.

Um zu beweisen, dass die tonische Kontraktion durch eine nervöse Erregung, und nicht durch Fehlerquellen irgendeiner Art hervorgerufen wird, wurde folgender Versuch vorgenommen: Der Ischiadicus des gefesselten Beines wurde freigelegt, mit versenkbaren Elektroden armiert und wieder ins Muskelfach eingelegt. Hierauf wurde eine tonische Kontraktion durch Kochsalzinjelktion in der beschriebenen. Weise (mit Massage) hervorgerufen und dann ein konstanter Strom durch die Elektroden in den Nerven eingeschlichen, und zwar so, dass die Anode dem Muskel am nächsten lag. Bei 
dem Einschleichen ging die Kurvegegen das Normalniveau zurück, um beim Ausschleichen wieder beträchtlich in die Höhe zu gehen. Die Kurve 8 gibt das Resultat eines solchen Versuchs graphisch wieder.

Kurve 8 (Taf. II.) (14. Mai 1905). Temporaria. Bei $\mathrm{NaCl}$. wird $1 \mathrm{ccm} \mathrm{NaCl}$ $(10 \%)$ in den Rückenlymphsack mit einer Pravaz'schen Spritze eingeführt. $M$ : Massage. Bei $S_{1}$ : der Strom (6 Daniell) wird eingeschlichen, doch zu schnell; es entstehen kräftige Zuckungen. Anfang, Dauer des Einschleichens sowie Anfang, Dauer und relative Intensität des eingeschlichenen Stromes wird in dem Schema unter der Kurve angedeutet. Bei $R$ Vergrösserung des Widerstandes im Kreis. Bei $\ddot{O}_{1}$ wird der Strom wieder ausgeschlichen (Ansteigen des Tonusl. Bei $S_{2} \cdot$ wieder, und zwar in derselben Intensität, eingeschlichen. Bei $X X$ kleinere Variationen in der Grösse des Widerstandes, die Zuckungen hervorrufen. Bei $S_{3}$ wieder eingeschlichen; bei $S_{4}$ ziemlich plötzliche Verstärkung des Stromes (Absinken des Tonus ohne Zuckung). Bei $\ddot{O}_{3}$ ist der Strom wieder ganz ausgeschlichen. Die Zuckung bei $Z$ tritt bei Öffnung des Stromkreises ein.

Es ist mir leider nicht möglich gewesen, eine Form von unpolarisierbaren Elektroden zu finden, welche unter den gegebenen Bedingungen leicht applikabel wäre. - Die Senkung nach der Zuckung bei $Z$ wird durch die Zuckung hervorgerufen; darüber später.

Ein ähnliches Resultat habe ich bei Experimenten erhalten, wo der Tonushochstand in anderer Weise hervorgerufen wurde.

\section{c) Einfluss der Dehnung auf das Zustandekommen tonischer Kontraktionen.}

1. Gedehnte Muskeln sind für die Wirkung tonischer Reize erheblich empfindlicher als Muskeln, welche ihre normale Länge besitzen. (Das Niveau, auf dem die schreibende Spitze steht, wenn der Muskel nicht gedehnt und nicht künstlich in kontrahierten Zustand versetzt worden ist, bezeichne ich, der Kürze halber, als Normalniveau.) Die Muskeln kontrahieren sich dann mit Belastung erheblich stärker als bei der gewöhnlichen Belastung und natürlichen Länge. Andauernde Dehnung des Muskels ist nicht günstig.

Kurve 12 (Taf. II.) (7. Mai 1905). Temporaria. Vor B el 30 wird mehrmals versucht, durch faradische Reizung der Rückenhaut von einer bestimmten Stromintensität tonische Kontraktionen hervorzurufen; doch ohne Resultat. Bei Bel 30 wird der Muskel mit etwa 2,1 $\mathrm{g}$ belastet (30 $\mathrm{g}$ an der Achse) und dehnt sich. 1,2,3 elektrische Hautreize derselben Intensität an derselben Stelle appliziert. Bei $L$ os wird das Extragewicht abgehoben; die Spitze geht 
erst sehr schnell, dann erheblich langsamer gegen das Normalniveau zurück. Bei 4 noch einmal derselbe Reiz; der Muskel geht dabei über sein normales Niveau hinaus und kehrt dann ziemlich genau dazu zurück. Bei 5 ist derselbe Reiz wieder unwirksam.

2. Wenn ein Muskel, wie im vorigen Versuch, gedehnt wird und das Gewicht wieder losgelassen wird, geht der Muskel nicht ohne weiteres auf seine alte Länge zurück. Es bleibt ein Dehnungsrückstand, der erst sehr langsam verschwindet. Wenn den Tieren in diesem Stadium schwache Reize zugeführt werden, wie z. B. elektrische Hautreize, so geht der Muskel plötzlich auf seine Normallänge zurück, jedoch ohne Zuckung. (Reizt man beim Nervmuskelpräparat den Nerven nach Dehnung des Muskels mit genau abgestuften Einzelschlägen oder Wechselströmen, so geht der Muskel, wenn überhaupt eine Reaktion eintritt, nur unter Zuckung zum Normalniveau zurück, aber nie direkt.) Wenn der Reiz ziemlich schwach gewesen ist, geht der Muskel nur ein kleineres Stück zurück und setzt dann nach dem Aufhören des Reizes etwa ebenso langsam wie vorhin sein Zurückgehen auf die Normallänge fort. Ist der Reiz dagegen stark oder der Muskel seiner normalen Länge schon ziemlich nahegekommen, so geht die Spitze über das Normalniveau in einer tonischen Kontraktion hinauf, kehrt aber zu diesem (ziemlich langsam) wieder zurück, nachdem der Reiz aufgehört hat.

Kurve 12 und 13 (Taf. II.) (7. Mai 1905, 9. Mai 1905). In Kurve 13 bedeutet $B$ e $l 50$ jedesmal die Belastung des Muskels mit $3^{1 / 2} \mathrm{~g}, L$ os jedesmal das Abheben dieses Gewichtes; $E_{1} \ldots E 6$ elektrische Hautreize. Bei $E 6$ geht die Kurve über das Normalniveau hinauf; senkt sich dann wieder gegen dasselbe. Das letztgenannte Phänomen ist auch in der Kurve 12, Reiz $4 \mathrm{zu}$ sehen.

\section{d) Literatur.}

Über tonische Kontraktionen bei Fröschen war in der Literatur sehr wenig zu finden. Aus der von Eckhard ${ }^{1}$ ) gelieferten Geschichte der Entwicklung der Lehre von den Reflexerscheinungen geht aber hervor, dass die älteren Autoren diese Kontraktionen wohl kaum beobachtet haben. Man findet nur Angaben über die Einwirkung von verschiedenen Temperaturen auf die schnellen Zuckungen.

1) C. Eckhard, Geschichte der Entwicklung der Lehre von den Reflexerscheinungen. Beiträge zur Anatomie und Physiologie Bd. 9 S. 29-192; speziell 135-139. 
So haben Cayrade ${ }^{1}$ ), Tarchanow ${ }^{2}$ ) und P. Archangelsky ${ }^{3}$ ) eine Steigerung der Reflexe nach Erwärmung gefunden, eine Steigerung, die aber um so schneller einer Depression Platz machte, je höher die Temperatur wurde - eine Beobachtung, die ich nur bestätigen kann. Tarchanow ${ }^{2}$ ) und $\mathrm{Wundt}^{4}$ ) haben Angaben über die Änderung in der Länge der Zuckung und der Latenzzeit bei Anwendung von Kälte gebracht. Freusberg ${ }^{5}$ ), der die Angabe von Tarchanow bestätigte, dass nach Iokaler Kälteeinwirkung auf die Haut die schnellen Reflexe gesteigert werden, erklärte dies so, dass die Applikation von Kältereizen auf die Haut erregbarkeitserhöhend auf das Zentrum wirkt. Er konnte dasselbe Resultat nämlich durch Wärmereizung der Haut bekommen. Endlich hat Biedermann ${ }^{6}$ ) in einer Arbeit aus dem Jahre 1900 zuerst die Aufmerksamkeit darauf gelenkt, dass das Rückenmark (S. 417) "bei irgend erheblicher Ablüblung in einen Zustand gerät, in welchem. es sich ausserordentlich geneigt zeigt, selbst die flüchtigsten Reize mit lang andauernder tonischer Erregung zu beantworten, ja, wie es scheint, in einzelnen Fällen auch ohne Hinzukommen eines nachweisbaren äusseren Reizes, während langer Zeit ununterbrochen auszusenden". Es liess sich auch zeigen, dass, wenn auch fast alle jene Reaktionen bei Fröschen fehlten, die sonst durch Sinnesreize unter Vermittlung des Gehirns hervorgerufen werden, die Anspruchsfähigkeit des Rückenmarkes erheblich gesteigert war. Es können Reflexzuckungen durch Reizung eines peripheren Nerven erhalten werden; jede von diesen Reflexzuckungen zeigt mehr oder weniger ausgeprägt einen tetanischen Verlauf ("was schon von $\mathrm{Cy}$ on be-

1) J. Cayrade, Recherches cutiques et expérimentales sur les mouvements. réflexes p. 48 ff. (Zit. nach Eckhard S. 185.) Paris 1864.

2) Tarchanow, Über die Wirkung der Erwärmung resp. Erkältung auf die sensiblen Nerven usw. Bull. de l'Acad. des Sciences de St. Petersbourg. t. 16 p. 223.1871 . (Zit. nach Eckhard S. 137.)

3) P، Archangelsky, Über den Einfluss der Wärme auf das Nerven- und Blutgefässsystem. Militärärztliches Journal. Februarheft S. 27. Russisch. St. Petersburg 1873. (Zit. nach Eckhard S. 136.)

4) W. Wundt, Untersuchungen zur Mechanik der Nerven- und Nervenzentren. II. S. 56 . Stuttgart 1876.

5) A. Freusberg, Über die Erregung und Hemmung der Tätigkeit der nervösen Zentralorgane. Pflüger's Arch. Bd. 10 S. 174-208, S. 181.1875.

6) W. Biedermann, Beiträge zur Kenntnis der Reflexfunktion des Rückenmarkes. Pflüger's Areh. Bd. 80 S. 408-469. 1900. 
obachtet worden ist"). Leider hat der Verfasser als Demonstration von diesem interessanten Befund keine Kurven seiner Arbeit mitgegeben. Es ist mir daher nicht möglich, festzustellen, inwiefern diese Kontraktionen mit den von mir gefundenen tonischen Kontraktionen zu identifizieren sind. Da die von mir beobachteten Kontraktionen nur sehr selten die Höhe einer schnellen Zuckung erreichen und noch weniger die Höhe eines Tetanus, so scheint die Identität jedenfalls nicht sehr wahrscbeinlich. - Als Folge von Faradisation des Ischiadicus der anderen Seite und bei Hautreizen hat aber Beaunis ${ }^{1}$ ) (ohne dass ich bei meinen Versuchen davon wusste) bereits tonische Kontraktionen gesehen, auch bei unvergifteten Tieren und normaler Temperatur. Dagegen liegen bei Säugetieren und Wirbellosen eine grössere Anzahl von Beobachtungen über Tonuskontraktionen vor (Bubnoff und Heidenhain ${ }^{2}$, Sherrington ${ }^{3}$ ), Uexküll $1^{4}$ ), Jordan usw.]. Bubnoff und Heidenhain haben Tonuskurven bei direkter Reizung der weissen, resp. grauen Substanz des Gehirns bei Warmblütern erhalten. Die von der Rinde ausgelösten Zuckungen waren erheblich länger als die von der weissen Substanz. Sherrington beschreibt als "eataleptoid reflexes" ausserordentlich langdauernde und langsame Kontraktionen auf kurze Reize hin, die er bei Affen nach Entfernung des Grosshirns beobachtet hat.

1) H. Beaunis, Recherches experimentales sur les conditions de l'activité cérébrale etc. I. p. 95. Paris 1884.

2) N. Bubnoff und R. Heidenhain, Über Erregungs- und Hemmungsvorgänge innerhalb der motorischen Hirnzentren. Pflüger's Arch. Bd. 26 S. 137-200 speziell Taf. IV. u. V. 1881.

3) C. S. Sherrington, Cataleptoid reflexes in the monkey. Proc. Roy. Society vol. 60 p. $411-414$. 1897. - On the innervation of antagonistic muscles. Sixth Note. Proc. Roy. Soc. vol, 66 p. 66-68. 1900. - On reciprocal innervation of antagonistic muscles. Seventh Note. Proc. Roy. Soc. vol. 76 B p. 269 -278. 1905. - Decerebrate rigidity and reflex coordination of movements. Journal of Physiology vol. 22 p. 319-332.

4) J. v. Uexküll, Studien über den Tonus. I. Zeitschr. f. Biol. Bd. 44 S. 269-344. 1903. - Studien über den Tonus. II. Zeitschr. f. Biol. Bd. 46. 1905. Studien über den Tonus. III. Zeitschr. f. Biol. Bd. 46. 1905. - Leitfaden in das Studium der experimentellen Biologie der Wassertiere. Wiesbaden 1905. 
C. Die Beeinflussung der tonischen Zustände und der direkten Erregbarkeit des Nerven durch dynamogene und inhibitorische Reize.

\section{Methodisches.}

In den hier mitgeilten Versuchen über die Wirkung von hemmenden Reizen wurden die Tiere so gefesselt, wie bei den Untersuchungen über die tonischen Kontraktionen. Die Vorrichtungen, um die Temperatur konstant $z \mathfrak{u}$ erhalten, waren auch dieselben. Die wichtigste Komplikation bestand darin, dass der Nervus ischiadicus des gefesselten Beines rhythmisch jede zweite Sekunde mit Öffnungsinduktionsschlägen von eben wirksamer Stärke gereizt wurde. (Über die dabei zu benützenden Kautelen siehe unten im Kapitel: Schwellenwanderung und Sehwellenschwankung.). Als Stromquelle dienten dabei 4-6 Daniels. Die Unterbrechungen geschahen durch ein Metronom; bei diesem war Alkoholspülung der Quecksilberoberflächen, um eine konstante Schlagstärke zu bekommen, unbedingt nötig. Zur Abblendung der Schliessungsschläge diente ein von Herrn Professor Dr. J. R. Ewald konstruierter Apparat, bei dem auch die unipolaren Wirkungen der Schliessungsschläge ausgeschaltet sind. (Die Beschreibung des Apparates wird durch Herrrn Professor Dr. Ewald selbst erfolgen.) Die Induktionsschläge wurden vermittelst versenkbarer Elektroden dem Nerven zugeführt. Die Furche in der einen Hartgummiplatte derselben war so klein, dass der Nerv eben gerade hineinpasste. Schon hierdurch wurde eine Veränderung der Lage der Elektroden auf dem Nerven fast vollkommen vermieden. Um derartige Verschiebungen vollständig auszuschliessen, wurde eine Gelatine hergestellt, deren Schmelzpunkt etwas höher lag als die Zimmertemperatur. Ein wenig noch halbflüssiger Gelatine wurde dann auf die nicht mit Elektroden armierte Hartgummiplatte der. versenkbaren Elektroden gebracht; der Nerv wurde in die Furche eingelegt (und hatte also auf der einen Seite die Platindrähte, auf der anderen die Gelatine), und es wurde gewartet, bis die Gelatine erstarrte: der Nerv war so unbeweglich befestigt. Zerren an den Elektroden änderte dann nichts an dem Reizerfolg.

Um Hemmungen hervorzurufen wurde in vielen Experimenten der Ischiadicus der rechten Seite mit faradischen Strömen gereizt. Es wurden dabei ebenfalls versenkbare Elektroden gebraucht. 


\section{Schwellenschwankung und Schwellenwandernng.}

Um die oben unter I. angegebene „eben wirksame" Schlagstärke zu finden, muss man auf gewisse, bisher weniger beachtete Bedingungen der Nervenreizung achtgeben.

Beim Herausprobieren der Schwelle des peripheren Nerven geht man ja gewöhnlicherweise von unwirksamen Reizen langsam aufwärts, bis man sie gefunden hat. Es zeigt sich dann wohl immer, dass es, auch bei Konstanz des Schlages und wenn der Nerv gut feucht gehalten wird, eine gewisse Stärkeordnung gibt, bei welcher der Muskel sich das eine Mal kontrahiert, das andere Mal aber nicht. Gewöhnlicherweise braucht man kaum mehr als um ein paar Zentimeter den Rollenabstand zu verkleinern, um konstante Zuckungshöhen zu bekommen; schon bei etwas kleinerer Reizintensität reagiert der Muskel jedesmal mit einer Zuckung, nur ist die Höhe ziemlich variabel. Steht der Nerv noch mit dem Rückenmarke in Verbindung (kein Reflexpräparat!), so fällt einem die relativ breite Zone des nicht sicheren Reagierens auf. Besonders auffallend werden die Variationen in der Reizschwelle, wenn das Tier sich bewegt oder Unruhe zeigt. Bei abgekühlten Tieren ist dasselbe in noch böherem Grade der Fall. Speziell ist hier die Zone von konstantem Reagieren, aber mit inkonstanter Zuckungshöhe auffallend gross. Benützt man nun ein Tier mit durchschnittenen hinteren Wurzeln, so treten diese Phänomene nicht so auffallend auf, sind aber immer vorbanden. Durchschneidet man den Ischiadicus hoch und reizt das periphere Ende, so werden sie noch weiter reduziert.

Reizt man den Nerven (gleichgültig ob mit dem Zentrum in Verbindung stehend oder nicht) längere Zeit mit der Reizstärke, welche zunächst als Schwelle für konstante Zuckungshöhe gefunden wurde, so kann man oft den Rollenabstand um mehrere Zentimeter vergrössern, ohne dass die Zuckungen aufhören, konstant zu bleiben, geschweige denn, dass Ausfälle auftreten. Macht man jetzt eine Pause von 1-2 Minuten, so findet man die zuletzt angewandte Schlagstärke entweder unwirksam oder von inkonstantem Erfolg, und man muss zur Erreichung gleichmässiger Zuckungshöhe wieder zur Anfangsschwelle zurückgehen. Dieses Herunterdrücken der Schwelle durch Reizung nenne ich Schwellenwanderung, und im Gegensatz dazu bezeichne ich als Schwellenschwankung die Unregelmässigkeiten im Reizerfolg (variable Zuckungshöhe, resp. voll- 
kommenes Ausbleiben), welche bei geringerer Reiżintensität, als dem Schwellenreiz für konstante Zuckungshöhe entspricht, eintreten.

Vor allem wirkt die Kälte auf die Schwellenwanderung fördernd. - Auch die Frequenz der Reizung spielt bei der Entstehung dieser Phänomene insofern eine Rolle, als bei sehr grosser Frequenz die Schwellenschwankung bald aufhört, um der Schwellenwanderung Platz zu machen; bei geringerer Frequenz verschwindet die Schwellenwanderung nicht, aber sie wird geringer. Die Schwellenschwankung kann bei geringerer Frequenz kleiner werden; es wurde aber auch Vergrösserung der Schwankung in verschiedenen Fällen beobachtet. Von weit geringerer Bedeutung für das Eintreten der Schwellenwanderung als die Frequenz scheint die Schlagstärke zu sein. Es wurde mehrmals versucht, durch eine Reihe von starken Schlägen ( 0 bis 5 cm Rollenabstand eines grossen Induktoriums) die Schwellenwanderung schnell hervorzurufen, doch war die Veränderung weder konstant noch gross. Wenn die Wanderung ein paarmal durch längere Reihen von Schlägen hervorgerufen worden ist, tritt sie nachher nicht mehr so leicht ein, und sie wird auch nicht mehr sehr gross. Ich teile nun hier einen Teil vom Protokoll über einen Versuch rom 15. April 1905, in dem man Schwellenschwankung und Schwellenwanderung findet, mit. Die Wanderung erstreckt sich in diesem Fall, wo die hinteren Wurzeln intakt waren, das Tier relativ stark tonisch reagierte (nach längerer Abkühlung) und der Versuch bei ziemlich niedriger Temperatur (plus $12^{\circ}$ C.) stattfand, auf $7 \mathrm{~cm}$ Rollenabstand, einen Wert, den man jedoch relativ selten findet. Bei Nervmuskelpräparaten kann sie unter allen Kautelen, meinen Erfahrungen nach, eine Grösse von höchstens $3 \mathrm{~cm}$ erreichen. (Die Erscheinung kann nicht etwa auf Verschiebung der Elektroden, welche übrigens technisch ausgeschlossen war, zurückgeführt werden, denn die Erregbarkeit wächst.)

(Siehe die Tabelle auf S. 268 und 269.)

Um der wirklichen Schwelle nahe zu kommen, wurde darum meistens folgendermassen gearbeitet: Von unten kommend wurde der Rollenabstand, bei dem die Zuckungen, allen Bewegungen des Tieres zum Trotz, konstant waren, festgestellt. Dieser Abstand wurde dann um 3 , in Fällen von starkem Tonus um $4 \mathrm{~cm}$ allmählich vergrössert. Es dauerte selten mehr wie 2 Minuten, bis der Muskel eine vollkommen konstante Zuckungsserie schrieb. 
Über ein ähnliches Verhalten des Muskelnerven bei Tieren, "resté longtemps inactifs", hat M. T. Guilloz in Comptes Rend. de la société biol., 13 juin 1904, Mitteilung gemacht. Dasselbe Phänomen ist auch von $\mathrm{Harless}^{1}$ ) schon 1858 beschrieben worden. Es liegen auch von Beauni ${ }^{2}$ ) Beobachtungen vor, die zeigen, dass bei der Reizung eines Nerven mit rhythmischen Einzelschlägen noch etwas anderes geschieht, als dass sich nur die Erregungen mit einer gewissen Geschwindigkeit zum Muskel fortpflanzen; zwischen den Einzelschlägen befindet sich der gereizte Nerv nicht in dem Ruhezustand des ungereizten Nervens.

\section{Tabelle.}

Schwellenschwankung und Schwellenwanderung.

Versuch vom 15. April 1905. Grosse Esculenta, ohne Wurzeloperation.

Versenkbare Elektroden.

\begin{tabular}{|c|c|c|c|}
\hline $\begin{array}{c}\text { Rollen- } \\
\text { abstand } \\
\text { (grosses } \\
\text { Induktorium) }\end{array}$ & $\begin{array}{l}\text { Zahl der } \\
\text { Öffnungs- } \\
\text { schläge, } \\
\text { welche dem } \\
\text { Nerven zuge- } \\
\text { führtwurden }\end{array}$ & $\begin{array}{c}\text { Zahl der } \\
\text { wirksamen } \\
\text { Schläge }\end{array}$ & Bemerkungen \\
\hline $\begin{array}{c}\text { bis 22 unwirksam; } \\
\text { nicht graphisch } \\
\text { registriert } \\
22 \\
21 \\
20 \\
19 \\
18 \\
\\
17 \mathrm{a} \\
17 \mathrm{~b} \\
18 \\
19 \\
20 \\
21 \\
22 \\
23 \mathrm{a} \\
23 \mathrm{~b} \\
23 \mathrm{c} \\
23 \mathrm{~d} \\
24 \\
25 \\
26 \\
27\end{array}$ & $\begin{array}{r}15 \\
15 \\
15 \\
15 \\
50 \\
\\
23 \\
60 \\
20 \\
65 \\
190 \\
25 \\
20 \\
20 \\
20 \\
20 \\
18 \\
20 \\
90 \\
65 \\
110\end{array}$ & $\begin{array}{r}0 \\
1 \\
0 \\
0 \\
35 \\
\\
22 \\
60 \\
19 \\
29 \\
149 \\
25 \\
20 \\
5 \\
7 \\
19 \\
18 \\
20 \\
74 \\
52 \\
67\end{array}$ & $\begin{array}{l}\text { Beim Einstellen. } \\
\text { Im Anfang ein paar Zuckungen } \\
\text { ausserhalb des Rhythmus. Das } \\
\text { Tier ist unruhig. } \\
\text { Unmittelbare Fortsetzung von } 17 \text { a. } \\
\text { So geordnet, dass sich immer } \\
\text { grössereGruppen von Zuckungen } \\
\text { bilden mit immer kleineren Aus- } \\
\text { fällen dazwischen Dieletzten } 40 \\
\text { konstant wirksam. } \\
\quad \\
\text { Regelmässig verteilt. } \\
\text { Sehr "unregelmässig verteilt. }\end{array}$ \\
\hline
\end{tabular}

1) E. Harless, Molekulare Vorgänge in der Nervensubstanz. I. S. 6 u. 40. München 1858.

2) H. Beaunis, l. c. S. $95-100$. 


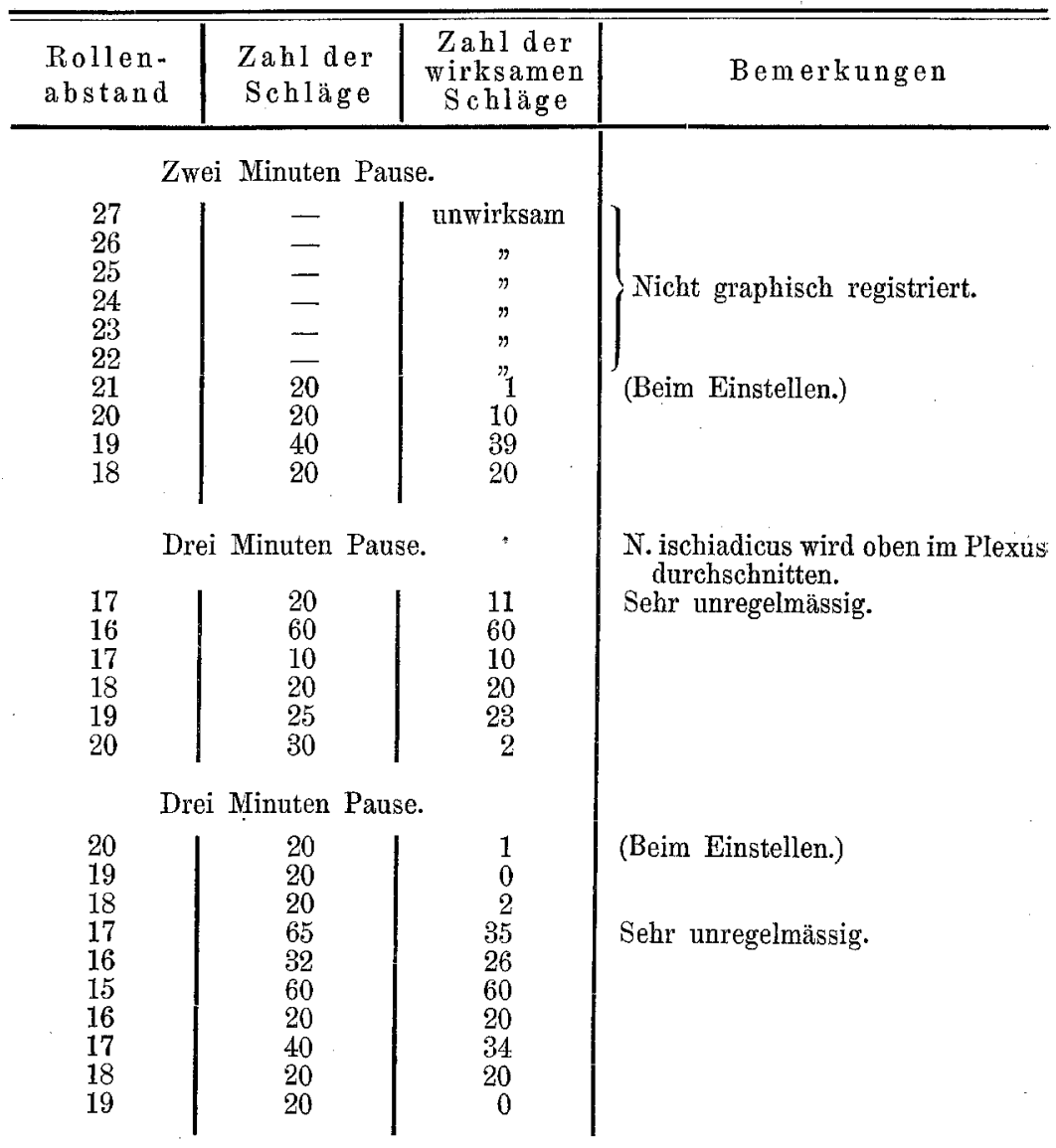

\section{Mittel, Hemmungen hervorzurufen.}

Um dem Zentralnervensystem hemmende Reize zuzuführen, wurden zwei verschiedene Methoden benützt.

1. Die eine war die alte, schon seit $\mathrm{Herzen}^{1}$ ) benutzte Methode der Faradisation des Ischiadicus der einen Seite. Von mehreren der Forscher, die diese Methode benutzt haben, unter anderen Nothnagel ${ }^{2}$ ), Biedermann ${ }^{3}$ ), Verworn ${ }^{4}$ ), wird

1) Herzen, Experiences sur les centres modérateurs de l'action reflexe. Turin 1864 (zit. nach Goltz, Beiträge usw. S. 48. Berlin 1869).

2) H. Nothnagel, Bewegungshemmende Mechanismen im Rückenmark des Frosches. Zentr. f. d. med. Wiss. S. 211 u. 212.1869.

3) W. Biedermann, l. c.

4) M. Verworn, Zur Physiologie der nervösen Hemmungserscheinungen. Arch. f. Phys. Suppl. S. 105-124. 1900. 
die Unsicherheit der Methode unter gewöhnlichen Umständen betont, und ich kann ihnen nur beistimmen. Dagegen ist nach Biedermann ${ }^{1}$ ) das Resultat bei Kältetieren konstanter, und auch für den nicht dekapitierten Kältefrosch stimmt dies. Bei Wärmetieren und normalen Tieren ist die Wirkung nach meinen Erfahrungen sehr launenhaft; das Misslingen ist sehr gewöhnlich, und die Unruhe des Tieres vor und nach der Tetanisation, besonders bei Wärmetieren, sehr gross, wodurch die Schwierigkeiten der Feststellung des eben wirksamen Induktionsschlages grösser wie sonst werden. Bei Kältetieren dagegen tritt in fast allen Fällen wenigstens vollständige Ruhe des Tieres während der. Faradisation ein, und sehr oft ist die Hemmung so stark, dass sogar die als Index für die starke Hemmung gebrauchten Kornealreflexe wegfallen.

2. Sowohl für Kälte- wie für Wärmetiere gibt es aber ein sehr gutes Mittel, Hemmungen hervorzurufen, das Bethe ${ }^{2}$ ) zuerst beschrieben hat: die Hemmung durch Kochsalzinjektion in den Rückenlymphsack. Injiziert man einem Frosch etwa $1 \mathrm{ccm}$ einer mehrprozentigen Kochsalzlösung (in meinen Versuchen immer 10\%) in den Rücken- resp. Bauchlymphsack, so tritt bald (nach Massage der zugehörigen Haut besonders schnell) eine ausserordentlich starke Hemmung ein. Die Muskulatur wird schlaff, das Tier zieht beim Kneifen des Beines dasselbe nicht mehr an; die Kornealreflexe sowie alle anderen Reflexe hören auf, die Atmung steht still, das Tier lässt sich jede Lage — auch die Rückenlage - gefallen und lässt sich während mehrerer Minuten auch durch sehr energische Reize nicht aus diesem Zustande wecken. Nach und nach verliert sich aber die Intensität der Hemmung; gewöhnlicherweise kehren zuerst die Kornealreflexe mit wachsender Intensität wieder; einzelne Atemzüge treten auf und werden häufiger; schliesslich werden auch die Augenlider wieder bewegt. Liegt das Tier auf dem Rücken, so kann es noch mehrere Minuten in derselben Stellung bleiben. Gewöhnlich wird jedoch der Kopf etwas gehoben, einzelne schwache und unkoordinierte Bewegungen treten auf, besonders in den vorderen Gliedmassen. Während der ganzen Zeit tiefer Hemmung beobachtet man nicht selten ein Flimmern der Muskulatur, besonders des Ober-

1) W. Biedermann, l. c.

2) A. Bethe, Allgemeine Anatomie und Physiologie des Nervensystems S. 376. Leipzig 1903. 
schenkels; sowie die ersten eben beschriebenen Anzeichen des Erwachens auftreten, hört dieses Flimmern auf. Noch einige Augenblicke, und das Tier dreht sich mit sichtbarer Anstrengung um, um dann (scheinbar plötzlich wieder im Besitz seiner vollen Bewegungsfähigkeit) in langen und ausserordentlich energischen Sprüngen wegzulaufen. Liegen die Tiere nicht auf dem Rücken, so scheint die Wirkung schneller vorüberzugehen. Dieses Umdrehen kommt schneller und wird erleichtert durch irgendeinen peripheren Reiz, z. B. Kneifen einer Zehe des Hinterbeines; oft genügt sogar ein Anblasen. Durch eine neue Massage lässt sich in der Regel die tiefe Henımung wieder herstellen, doch dauert sie diesmal nicht mehr so lang.

Wenn das Tier nach dem Erwachen einige von den ohen erwähnten starken Sprüngen gemacht hat, bleibt es plötzlich wieder wie tot liegen (mit ausgestreckten Beinen) und erweist sich als reflektorisch vollkommen unerregbar. Lässt man das Tier in Ruhe, so wacht es bald wieder auf und setzt die schnelle, energische Flucht fort, fällt eventuell nach noch einigen Sprüngen wieder gehemmt und reflexlos zusammen, um dann nach einem ganz kurzen Intervalle, das durch periphere Reize sehr stark verkürzt werden kann, wieder in der Flucht die Rettung zu suchen. Dass die Tiere ein drittes Mal gehemmt werden, ist nicht sehr ungewöhnlich, mehr habe ich nur selten beobachten können. Lässt man sie aber nach der schnellen Umdrehung aus der Rückenlage nicht weglaufen, sondern setzt sie z. B. in ein Glasgefäss, genügend gross, um Bewegungen zu erlauben, und lässt sie in Ruhe, so bewegen sie sich wohl etwas, bleiben aber fast gleich rubig sitzen, und eine nochmalige Hemmung tritt unter solchen Verhältnissen fast niemals ein.

Sowohl Kalt- wie Warmtiere zeigen nach der eigentlichen tiefen Hemmung oft noch lange nachher eine Nachwirkung. Wenn man sie nämlich "hypnotisiert", durch eine plötzliche Umdrehung, bleiben sie entschieden leichter liegen wie vorher nicht gehemmte Tiere, und können dann 20 Minuten bis eine halbe Stunde liegen bleiben, wenn ihnen keine Reize zugeführt werden, was ein normaler hypnotisierter Frosch nur äusserst selten tut.

Ich habe verschiedene Versuche bei ungefesselten Tieren ausgeführt, um die Bedingungen dieser starken Hemmungen näher kennen zu lernen. Dass es sich nicht um einen Hautreiz handelt, scheint mir daraus hervorzugehen, dass Einreiben der Haut mit $10 \%$ iger Kochsalzlösung keine Hemmung hervorruft. Dass es sich 
auch nicht um eine Kochsalzzufuhr zum Zentralnervensystem handelt, scheint mir aus folgenden Erfahrungen hervorzugehen. Die kleinste Menge $10 \%$ iger Kochsalzlösung, mit der man ziemlich sichere Hemmungen (bei Kältetieren) hervorrufen kann, betrug bei meinen Versuchen $0,3 \mathrm{ccm}$. Wenn man diese Menge einem Kaltfrosch in die grosse Bauchvene injiziert, tritt kein Zeichen von Hemmung ein, geschweige dann einer plötzlichen; die Tiere reagieren vielmehr noch etwa 10 Minuten dem Äusseren nach wie sonst; dann tritt bei den meisten eine Senkung der Erregbarkeit ein, und binnen kurzem gehen die Tiere in einen schläfrigen Zustand über, der erst nach mehreren Stunden wieder verschwindet. Die wahrscheinlichste Erklärung der Hemmungswirkung dürfte wohl die sein, dass die grossen, durch den Rückenlymphsack gehenden Nervenstämme direkt gereizt werden. - Ganz auffallend ist die Wirkung auf das Herz; bei einem nach Engelmann suspendierten Herz konnte ich einen 15-20 Sekunden langen Stillstand beobachten, worauf die Kontraktionen allmählich wiederkamen. - Auch mit anderen Mitteln als mit Kochsalzlösung habe ich versucht, Hemmungen hervorzurufen (verdünnte Schwefelsäure, Crotonöl, Terpentin, Kaliumchlorid), doch gaben sie alle schlechtere Resultate wie die Kochsalzlösung.

Die Wirkung scheint bei den Kältetieren etwas leichter $\mathrm{zu}$ kommen wie bei Tieren, die mehrere Tage bei plus 25 bis plus $28^{\circ}$ C. gehalten worden sind. Der Unterschied im Äusseren ist aber nicht sehr gross. Das Flimmern der Skelettmuskulatur ist bei Warmfröschen seltener; sie werden aus der Hemmung leichter geweckt; meistens kann man auch bei einem Warmfroseh leichter wie beim Kaltfrosch einen Versuch ausführen, der sehr an den Goltz'schen Quakversuch erinnert. Berührt man nämlich das Tier, wenn es auf dem Rücken liegt und schon Andeutungen zum Aufwachen gezeigt hat, auf der Bauchhaut oder noch besser oben in der Nähe des Kehlkopfes, so stösst es bei jeder Berührung ein langes Quaken aus. Auch dieses Quaken kann dureh festen Druck eines Fusses gehemmt werden.

\section{Hemmung und Förderung tonischer Kontraktionen.}

a) Eigene Versuche.

Schon oben habe ich angedeutet, dass Versuchstiere gelegentlich in einen Zustand geraten können, in welchem das Zentralnervensystem ausgesprochen tonisch reagiert. Die Tiere erinnern dak 
oft im äusseren Benehmen an die typischen Frösche, die Verworn ${ }^{1}$ ) (1897) beschrieben hat. Auch nach ganz schwachen Reizen, vor allem bei Massage der Rückenhaut, haben sie dann die Tendenz, mit stark gehobenem Rücken, Kopf nach unten, sich auf den gestreckten Hinterbeinen in die Höhe zu heben. Die Vorderbeine sind gewöhnlicherweise flektiert. Sie scheinen dabei weniger empfindlich für Lichtreize zu sein, wie es sonst bei normalen Fröschen der Fall ist. Wie ich auch bemerkt habe, steigt der Tonus des Gastrocnemius dabei sehr langsam, aber ausserordentlich stark, und gleichzeitig scheint die Erregbarkeit für reflektorisehe Reize etwas erböht, wobei die tonische Reaktion etwas vorwiegt. Zwischen diesem Zustand und dem Zustand des Frosches, der schon tagelang in Zimmertemperatur gewesen ist, kann man oft Zwischenformen finden, die dann im allgemeinen den Eindruck machen, als wären die tonischen Kontraktionen noch irgendwie, irgend wo gehemmt; sie treten gruppenweise auf, und dies gibt dann das oben angeführte Phänomen, wo innerhalb eines Experimentes die Reaktion von vorwiegend schneller zu vorwiegend tonischer übergeht, vice versa, und dann mehrmalige Schwankungen hin und her auftreten. Setzt man das Experiment genügend lange fort, siegt meistenteils die tonische Reaktion. Und dies um so schneller, je mehr Reize auf die afferenten Nervenbahnen appliziert werden.

Bei solchen Tieren habe ich folgende Beobachtungen gemacht:

1. Wenn durch irgendeinen peripheren Reiz eine schnelle Reflexkontraktion hervorgerufen worden ist, so geht die Kurve nicht gleich auf das Normalniveau zurück, sondern es bleibt eine manchmal recht grosse Dauerverkürzung, die dann entweder sehr langsam oder häufiger ziemlich plötzlich verschwindet. Das letztere ist besonders der Fall, wenn gleichzeitig Bewegungen in anderen Körperteilen des Tieres auftreten. Ist die tonische Reaktion sehr stark, so bleibt die Kurve dann und wann auch dauernd ein wenig über dem Normalniveau. - Seltener ist das folgende analoge Phänomen: Der Muskel bekommt nach der schnellen Zuckung allerdings gleich seine normale Länge zurück, gleich nachher fänt die Kurve aberzu steigen an und zeigt eine langsam verlaufende tonische Kontraktion. -

1) M. Verworn, Tonische Reflexe. Pflüger's Arch. Bd. 65 S. 63-80. 1897. 
Nach einer nochmaligen Reflexzuckung tritt dann das Phänomen wieder auf.

Kurve 1 (Taf. II.) (7. Mai 1905). Temporaria. Sensible Wurzeln links durchschnitten. Bei Ke Kneifen des Vorderbeines, Zuckungen und grosser Verkürzungsrückstand. Übrige Bezeichnongen in der Kurve unter Abt. 7 (dieses Kapitels).

Kurve 10 (Taf. II.) (23. Mai 1905). Temporaria. Kochsalzinjektion. Bei Ber Berührung der Rückenhaut; schnelle Zuckung mit folgender tonischer Kontraktion.

Kurve 7 (Taf. II.) (11. Mai 1905). Bei a reflektorischer Tetanus (Rückenhaut); bei $b$ schnelle Zuckung nach Kneifen des rechten Beines. Nachher tonische Kontraktion. Bei $c$ einzelne spontane tonische Kontraktionen.

2. Bei einem Tier, bei welchem die sensiblen Wurzeln auf der Seite, deren Gastrocnemius schreibt, intakt sind, gibt Faradisation der Oberschenkelbaut derselben Seite (resp. Faradisation des Ischiadicus) nach dem Tetanus, ausser den gewöbnlichen bekannten, auf mechanische Faktoren zurückzuführenden Verkürzungsrückstand (der auch beim Nervmuskelpräparat zu beobachten ist), eine Verkürzung, die wahrscheinlich durch tonische Erregung zu erklären sein dürfte.

Kurve 9 (Taf. II.) (17. Mai 1905). Temporaria. Ei Faradisation der Oberschenkelhaut des linken Beines. Verkürzungsrückstand.

Der betreffende Rückstand ist deswegen wahrscheinlich tonischer Natur, weil ich ihn bei nicht toniseh reagierenden Tieren vergebens gesucht habe; auch lässt er sich durch reflektorische Reizungen beeinflussen, wie aus der Kurve 14 bervorgeht.

Kurve 14 (Taf. II.) (7. Januar 1906). Esculenta. Kalttier (sensible Wurzeln intakt). $a_{1}$ bis $\alpha_{2}$ faradische Reizung des linken Ischiadicus. Nachher Verkürzungsrückstand. Dauer der Tetanisation des rechten Ischiadicus (R.-A. $=0 \mathrm{~cm}$, kleines Induktorium) in der Signallinie angegeben. Bei $b$, gleich nach dem Anfang der Reizung, verlängert sich der linke Muskel. (Geht jedoch nicht vollständig auf das Normalnivean zurück.)

3. Bei Tieren, bei denen die tonische Reaktionsweise noch deutlicher auftritt, so dass die ersten Anfänge zum langsamen Tonussteigen schon vorhanden sind, beobachtet man nicht selten folgendes: Bei dem langsamen Ansteigen des Tonus, der. durch periphere Reize, z. B. schwache Reibung der Rückenhaut, beschleunigt werden kann, tritt oft eine spontane schnelle Zuckung ein; nach der Zuckung geht dann die Kurve direkt auf das Normalniveau zurück, um nach einem Bruchteil einer Minute wieder anzufangen zu steigen. Wieder tritt dann eine spontane Kontraktion ein, die Kurve geht wieder auf das Normalniveau zurück usw. - Tritt während einer tonischen Kon- 
traktion eine reflektorische resp. spontane, schnelle Zuckung ein, (wozu die tonische Kontraktion zu disponieren scheint), so sinkt die Kurve nach der Zuckung wieder plötzlich auf das Normalniveau zurück. Häufig tritt dann ohne weiteren Reiz wieder eine langsam steigende, tonische Kontraktion ein, die dann oft auch von einer schnellen Zuckung gelöst wird usw.

Kurve 9 (Taf. II.) (17. Mai 1905). Temporaria. In dem tonischen Rückstand nach dem reflektorischen Tetanus treten erst Unruhe (Un) und dann (Sp) drei spontane Zuckungen ein. Nach $S p$ sinkt die Kurve jedesmal ein Stück gegen das Normalniveau.

Kurve 10 (Taf. II.) (23. Mai 1905). Temporaria. Kochsalzhemmung ist hervorgerufen worden. (Pinc. N. NaCl.) Ber Berührung der Rückenhaut. Sp "spontane" Zuckung, Tonusfall; nachher geht die Kurve wieder langsam in die Höhe.

4. Hat man bei einem Versuchstier (Kältefrosch) eine tonische Kontraktion hervorgerufen, z. B. durch die angegebene Kochsalzmethode oder durch die Faradisation des rechten Ischiadicus, so kann man oft durch eine nochmalige Zufuhr von tonischen Reizen, am besten in irgendeiner anderen Körpergegend, die Kurve zu noch grösserem Steigen veranlassen. Oft genügt auch eine mässige Steigerung der Stärke der Induktionsschläge, um die tonische Kontraktion noch stärker zu machen.

Kurve 32, 2 (Taf. IV.) (7. Dezember 1905). (Untere Reihe.) Grosse Esculenta, Kalttier. Von $\gamma_{1}$ bis $\delta$ Faradische Reizung des rechten Ischiadicus, mit von 2 bis 0 sinkendem Rollenabstand. Schwache tonische Wirkung. Von $\delta$ bis $\varepsilon$ Massage der Rückenhaut. Starke tonische Kontraktion.

5. Ist aber die tonische Kontraktion schon über den Höhepunkt fort, und hat das Sinken schon angefangen, so ist es nicht ungewöhnlich, dass neue Zufuhr von Reizen, statt, wie es auch der Fall sein kann, nochmalige Steigerung hervorzurufen, einen entschieden beschleunigten Abfall bewirkt. Nicht selten ist der Abfall ganz plötzlich. Nach Aufhören des Extrareizes steigt dann die Kurve in der Form einer tonischen Kontraktion häufig wieder in die Höbe.

Kurve 11 (Taf. II.) (7. Juni 1905). Esculenta. Starker Tonus nach Kochsalzinjektion. Bei $M$ Massage der Rückenhaut. (Man vergleiche auch Kurve 28 Taf. III.)

Kurve 14 (Taf. II.) (6. Januar 1906). Esculenta. Bei $a$ schwache Tetanisation des linken Ischiadicus. Bei $b$ Tetanisation des rechten Ischiadicus. Tetanus mit tonischem Rückstand, der durch die reflektorische Reizung gelöst wird.

6. Im Zusammenhang hiermit sei ein anderes Phänomen erwähnt, das ich ein paarmal beobachtet habe, doch erst seitdem 
meine Aufmerksamkeit durch eine interessante Arbeit von $\mathrm{Pari}^{1}$ ) darauf gelenkt wurde.

In der Deutung der Vorgänge gehen aber die Meinungen von Pari und mir auseinauder.

Pari untersuchte die Reflexfunktionen des Rückenmarkes stark abgekühlter, dekapitierter Frösche, bei denen der Ischiadicus der einen Seite mit Einzelschlägen gereizt wurde, und der Gastrocnemius der anderen Seite schrieb. 20-30 Minuten nach der Dekapitation erhielt Pari nur Reflexzuckungen; dagegen trat sofort nach der Dekapitation bei schwachen Reizen, statt Reflexzuckungen, eine reflektorische Verlängerung des Gastrocnemius ein. Pari deutet diesen Befund als Beweis für die Existenz aktiver reflektorischer Muskelverlängerung. Der Verfasser hebt ausdrucklich hervor, dass er nicht weiss, ob auch dieselben Verlängerungen bei nichttonischen Tieren vorkommen können, scheint aber, nach Hinweis auf die Arbeiten über Tonus von Heidenhain $(1856)^{2}$ ), Auerbach (1856) ${ }^{3}$ ) und Schwalbe $(1865)^{4}$ ), die sämtlich in ihrem Resultate negativ waren, $\mathrm{zu}$ meinen, dass die Verlängerungen vom tonischen Zustande unabhängig wären.

Ich habe nun ein paarmal ähnliche Phänomene beobachten können; doch soll gleich hervorgehoben werden, dass ich sie mehrmals vergebens bei Tieren gesucht habe, die nicht ausserordentlich stark tonisch reagierten. Auch ist es sehr selten gewesen, dass diese Verlängerungen einen so zuckungsähnlichen Charakter hatten, wie es in der Kurve Pari's der Fall ist. In der Tatsache hat aber Pari recht: ein Muskel kann reflektorisch verlängert werden; doch geht diese Verlängerung nach meinen Erfahrungen nie tiefer als bis auf die tonusfreie Länge. Ich werde weiter unten zeigen, dass eine sehr grosse Verlängerung eines Muskels durch reflektorische Reize hervorgerufen werden kann; nie habe ich aber eine Verlängerung über die tonusfreie Länge gefunden. Auch

1) G. A. Pari, Sull allungamento riflesso dei muscoli delle scheletro. Verworn's Zeitschr. Bd. 4 S. 127-140.

2) R. Heid en hain, Historisches und Experimentelles über den Muskeltonus. R. Heidenhain's Physiologische Untersuchungen S. 11-45. Berlin 1856.

3) Auerbach, Über den Muskeltonus. Jahresber. der schl. Gesellsch. f. vaterl. Kultur S. 127-130. 1856.

4) Schwalbe, Zur Lehre vom Muskeltonus. Unters. physiol. Laborat. zu Bonn. Herausgegeben von Pflüger. S. 64-79. Berlin 1865. 
Sherrington ${ }^{1}$, der neuerdings einen Muskel, der sich in Kontraktion befand, durch reflektorische Reize in der Kontraktion gehemmt hat, findet dabei, dass die Verlängerung ebensoweit geht; „even to postmortal length". Aber auch nicht darüber. Das Zurückgehen der Verlängerung hat nun denselben Charakter wie der Anfang von tonischen Kontraktionen, wie ich sie z. B. durch Berührung der Corneae hervorrufen konnte. (Schon oben habe ich ja auch auf die Empfindlichkeit der gedehnten Muskulatur für tonische Erregungen aufmerksam gemacht.) Unter solchen Umständen finde ich mich veranlasst, die $\mathrm{P}$ ari'sche Verlängerung als den durch einen schnellen Reiz hervorgerufenen Tonusfall aufufassen, der bei dem stark tonisch reagierenden Tiere gleich von einer neuen tonischen Kontraktion gefolgt wird.

7. Hat man in dem Gastrocnemius, z. B. bei einem stark tonisch reagierenden Tiere, durch eine Reflexzuckung eine tonische Nachkontraktion herbeigefübrt, und veranlasst man dann das Tier zu Innervation der Muskulatur des anderen Beines, z. B. durch Ausziehen desselben aus der Hockstellung, so tritt oft sogleich eine plötzliche Senkung des Tonus ein. Manchmal tritt mitten in diesem Abfall eine kleine Zuckung ein, wodurch der Abfall etwas beschleunigt wird. Der Abfall tritt, soviel ich weiss, aber nie ein, wenn das Tier bei dem Herausziehen des Beines keinen Widerstand leistet.

Kurve 1 (Taf. II.) (2. Mai 1905). Esculenta (reagiert stark tonisch). Bei $K e$ Kneifen des Vorderbeins; schnelle Zuckung mit starker Dauerverkürzung. Bei $B$ wird das rechte Hinterbein aus der Hockstellung ausgezogen. Das Tier leistet dabei einen Widerstand. Zurückgehen der Kurve auf das frühere Niveau.

\section{b) Literatur.}

Beobachtungen, die sich auf ähnliche Zustände beziehen, liegen

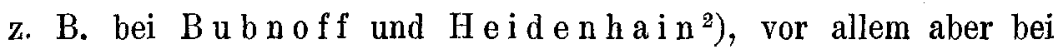
Sherrington vor.

$\mathrm{Bubnoff}$ und Heidenhain ${ }^{2}$ ) reizten bei Hunden in einem bestimmten Grade von Morphiumnarkose elektrisch und in verschiedenen Kombinationen das motorische Zentrum eines Körperteiles, z. B. einer Pfote, und die Haut derselben Gegend. Bei diesem

1) C. S. Sherringtan, on reciprocal innervation of antagonistic muscles. Seventh Note. Proc. Roy. Society vol. 76 B S. 269-278. 1905.

2) Bubn off und Heidenhain, l. c. 
bestimmten Grad der Narkose, dessen Auftreten durch starke Reizung des einen Ischiadicus beschleunigt werden konnte, fanden sie, dass alle Muskelaktionen der Tiere tonisch wurden. Die Beschreibung dieser Kontraktionen entspricht meinen Beschreibungen unter " $R$ egel der doppelten Möglichkeit". Verkürzungen, die durch Reizung der Rinde entstanden, gingen bei Wiederholung der Reizung leicht in tonische Kontraktur über. Wenn während einer solchen tonischen Kontraktion der Pfotenmuskulatur die Haut desselben Gliedes sanft gestrichen wurde, löste sich die tonische Kontraktion, meistens plötzlich, aber nicht immer vollständig. Ihre Versuchsergebnisse auf diesem Punkte fassen die Verfasser folgendermassen zusammen: schwache peripherische Reize mannigfachster Art sind also imstande, tonische Erregungen der motorischen Zentren aufzuheben; starke Reize rufen meistens eine tonische Kontraktion hervor. In einzelnen, seltenen Fällen wurde auch beobachtet, dass tonische Kontraktionen, die äusserst leicht entstanden, sich überhaupt nicht wieder lösen liessen. Weiter: die durch relativ starke Rindenreizung hervorgerufenen tonischen Kontraktionen konnten durch schwache Reize, auf dieselbe Stelle appliziert, gelöst werden. In gewissen Fällen konnte die Lösung auch durch Reize von bestimmten anderen Rindeteilen hervorgerufen werden.

C. S. Sherrington ${ }^{1}$ ) beobachtete nach Entfernung der Grosshirnhemisphären (bei verschiedenen Säugern) eine starke tonische Kontraktion der Extensoren der Gliedmassen; durch diese entsteht eine Steifheit in verschiedenen Gelenken, die als "decerebrate rigidity" bezeichnet wird. Diese starke tonische Kontraktion ist reflektorischer Natur, und zwar ist in der Hauptsache jedes Glied reflektorischer Erreger seiner eigenen Kontraktur. Sie kann durch Reizungen von verschiedenen Teilen des Zentralnervensystems aus gelöst werden und ebenso durch periphere Reizungen; Reflexe, die bei Tieren mit "decerebrate rigidity" beobachtet werden, zeigen immer Kontraktion in einer Muskelgruppe und gleichzeitig Hemmung in der Antagonistengruppe ("reciprocal innervation").

1) C. S. Sherrington, Cataleptoid reflexes in the monkey. Proc. Roy. Soc. vol. 60 p. $411-414$. 1897. - On the innervation of antagonistic muscles. Sixth Note. Proc. Roy. Soc. vol. 66 p. 66-68. 1900. - On reciprocal innervation of antagonistic muscles. Seventh Note. Proc. Roy. Soc. vol. 76B p. 269-278. 1905. - Decerebrate rigidity and reflex coordination of movements." Journal of Physiology vol. 22 p. $319-332$. 
M. Verworn ${ }^{1}$ ) fand bei seinen Untersuchungen über tonische Reflexe (1897), dass durch Reiben oder Druck der Seitenhaut des Rumpfes bei Rana temporaria (besonders nach Exstirpation des Grosshirns) auf reflektorischem Wege eine tonische Kontraktion der Muskeln in allen Körperteilen hervorgerufen wird, die den Reiz längere Zeit, bei grosshirnlosen Individuen unter Umständen eine Stunde, überdauert, so dass das Tier mit gekrümmtem Rücken in Katzenbuckelstellung auf gestreckten Extremitäten unbeweglich stehen bleibt. Andere als mechanische Reize besitzen nicht das Vermögen, den allgemeinen Reflextonus zu erzeugen. Eine Veränderung der Reflexerregbarkeit konnte der Verfasser in diesem Stadium nicht finden. Die Bahn der betreffenden Reflexe geht nach den Versuchen des Verfassers über die Ganglien in der Mittelhirnbasis und der Medulla oblongata.

Serien von starken tonischen Kontraktionen sind als "tonische Krämpfe" von verschiedenen Forschern beobachtet worden.

\section{Beeinflussung der Erregbarkeit des peripheren Nerven durch reflektorische, besonders hemmende Reize.}

Wenn man in oben angegebener Weise den linken Ischiadicus, dessen Gastrocnemius stets der schreibende Muskel war, rhythmisch mit Öffnungsschlägen so reizt, dass die Zuckungen konstante Höhe haben (siehe oben, Schwellenwanderung!), so können a uf reflektorische Reize hin Änderungen in der Zuckungshöhe hervorgerufen werden, sowohl positiver wie negativer Natur (Änderung der Anspruchsfähigkeit). Diese Änderungen der Anspruchsfähigkeit des Muskelnerven konnten hervorgerufen werden durch elektrische Reizung des anderen Ischiadicus, Hautreize, Lichtreize und $\mathrm{NaCl}$-Injektion in den Rückenlymphsack.

a) Reine Erregbarkeitsänderungen.

1. Reizt man unter Vermeidung der oben angegebenen Fehlerquellen den rechten Ichiadicus mit Einzelschlägen resp. ganz kurzen Serien von Wechselströmung, so beeinflussen diese reflektorischen Reize die Zuckungkurve, und zwar folgendermassen:

a) Wenn die Einzelschläge sehr stark sind, so verursachen sie zwischen den rhythmisch wiederkehrenden Zuckungen Reflexzuckungen.

1) M. Verworn, Tonische Reflexe. Pflüger's Arch. Bd.65 S. 63-80. 1897. 
b) Wenn sie nicht so stark sind, so zeigen sich an den rhythmischen Kontraktionen des linken Gastrocnemius Variationen in der Zuckungshöhe; ausserrhythmische Kontraktionen treten dann n i cht auf. Die Kontraktionen werden entweder grösser oder kleiner, oder es fällt eine ganze Zuckung aus.

Kurve 15 a (Taf. III.) (2. Dezember 1905). Esculenta (Kalttier). Ischiadicus rechts mit Schliessungs- und Öfinungsinduktionsschlägen gereizt. (Bei Schliessung geht der Hebel des Signals nach unten. Die Spitze des Signals ist um etwas mehr als $1 \mathrm{~mm}$ gegen die Spitze des Schreibhebels nach rückwärts verschoben.) Schlagstärke, Rollenabstand $=10 \mathrm{~cm}$, grosses Induktorium. Bei +++ ebenso viele Induktionsschläge, Vergrösserung zweier Zuckungen.

Kurve 15 b (Taf. 1II.) (ebenso). Bei + Öffnungsinduktionsschlag, mit verkleinerter Zuckung.

Wenn der Reiz kurz vor dem Anfang der Zuckung den Nerven trifft, so bewirkt er eine Erhöhung der Zuckung; trifft er den Nerven in dem Augenblick, wo sich der Muskel eben wieder vollständig verlängert hat, so bekommt man eine Senkung der Höhe der nächsten Zuckung. [Dies steht in guter Übereinstimmung mit den Befunden von Yerkes'); wenn zwei Reize auf ein Tier appliziert werden, ist die Grösse des Intervalles zwischen den beiden Reizen für die Wirkung bestimmend; bei Intervallen unter einem gewissen Wert tritt Babnung ein, bei grösseren Hemmung.]

2. Ausfälle (bei Kältetieren), resp. Vergrösserungen von Zuckungen, mit oder ohne einzelne Ausfälle (bei Wärmetieren), treten oft auch auf, wenn die Haut stark mechanisch gereizt wird (festes Anfassen mit einer Pinzette, Durchstechen der Haut mit der Nadel der PravazSpritze). Dasselbe Resultat wurde nach Betupfung der Corneae erhalten.

- Kurve 16(Taf.III.) (27. März 1905). Esculenta (Kalttier). Kochsalzhemmung soll hervorgerufen werden; zu diesem Zwecke wird die Rückenhaut mit einer starken Pinzette gefasst; Pine 1: beträchtliche Senkung von zwei Zuckungen. Das Tier ist dabei nicht unruhig. - Pinc 2: Die Haut wird wieder mit der Pinzette gefasst; eine Zuckung fällt aus. - Beschreibung der ganzen Kurve unter Abteilung 5 dieses Kapitels, S. 282.

3. Wenn ein Tier plötzlich stark beleuchtet resp. beschattet wird, entsteht ein Ausfall resp. starke Senkung der Höhe einer oder einiger Zuckungen.

1) R. M. Yerkes, Bahnung und Hemmung der Reaktionen auf taktile Reize durch akustische Reize beim Frosch. Pflüger's Arch. Bd. 107 S. 207 bis 238.1905 . 
Kurve 17 (Taf. III.) (29. Juni 1905). Temporaria. Bei $K 1, K 2, K 3$ plötzliche Beschattung; Ausfall von Zuckungen.

(Gelegentlich habe ich auch einen Ausfall beobachtet resp. absichtlich hervorrufen können, durch Bewegung von beleuchteten Flächen innerhalb des Gesichtsfeldes des Versuchstieres. Es ist deshalb bei allen derartigen Versuchen zweckmässig, wenn sich der Experimentator ausserhalb des Gesichtsfeldes des Frosehes befindet.)

4. Bei Unruhe des Versuchstieres (Bewegung in anderen Körperteilen) fallen oft einzelne Zuckungen aus resp. werden kleiner; auch können kürzere Reihen von Zuckungen wegfallen. (In seltenen Fällen tritt eine Steigerung der Anspruchsfähigkeit ein.) Meistens ändert sich die Zuckungshöhe $\mathrm{nach}$ einer solchen Unruheperiode, und zwar so, dass die Zuckungen, wenn sie vor der Unruhe gross waren, erheblich reduziert werden und vice versa.

Kurve 18 a (Taf. III.) (29. Juni 1905). Esculenta. $U 1 \ldots$ Unruhe des Tieres; Senkung der Zuckungshöhe. $U 2 \ldots$. Unruhe; Vergrösserung der Zuckungshöhe. Zuckungen, gross - Unruhe, klein - Unruhe, gross.

Kurve $18 \mathrm{~b}$ (Taf. III.) (ebenso). $U \ldots$. Unruhe; Zuckungen vor der Unruhe klein, nach der Unruhe gross.

5. Injiziert man einem Tier etwa $0,4-1 \mathrm{cem} K o \mathrm{chs}$ a l z $\mathrm{l}$ ös un $\mathrm{g}$ $\left(10^{\circ} \%\right)$ in den Rücken ly m phs a ck, so treten meistens sogleich, ohne Massage oder andere Reize, Ausfälle resp. Serien von Ausfällen auf (Kältetiere); Wärmetiere antworten in der Regel mit starken Vergrösserungen von einzelnen Zuckungen. Sind die Wärmetiere lange und stark warm gehalten worden, treten dann auch Zuckungen ausserhalb des Rhythmus auf.

Bei Versuchstieren, die eine Kochsalzinjektion bekommen haben, wird fast jeder nicht zu schwache Reiz Ursache zu Variationen in den Zuckungshöhen resp. zu Ausfällen. Besonders wirken in dieser Weise Massage der Rückenhaut und Licht, Reize, welche ohne vorhergehende Kochsalzinjektion wenig (Licht) oder gar nicht (Massage) erniedrigend auf die Zuckungen wirken.

Auch dabei antworten die Kältetiere in der Regel mit Ausfällen, Wärmetiere dagegen mit starken Vergrösserungen von einzelnen Zuckungen. Die Variationen in der Zuckungskurve treten nur währeind der allgemeinen Hemmung ein resp. während der Nachwirkung derselben. Vor dem Anfang der Hemmung wurden nie Ausfälle beobachtet. 
Kurve 16 (Taf. III.) (27. März 1905). Esculenta (Kalttier). Pine 1 und 2. Anfassen der Rückenhaut mit der Pinzette, Injektion $0,4 \mathrm{~cm} \mathrm{NaCl} 10 \%$. Hemmung tritt gleich ein und dauert so lange wie die Strichreihe. Senkungen der Höhe mehrerer Zuckungen. Massage 1 (Punktreihe): Ausfälle während und gleich nach der Massage. Massage 2: Ausfall gleich nach der Massage. Das Tier ist noch stark gehemmt. Massage 3: Ausfall gleich nach der Massage. Das Tier ist wenig gehemmt; gleich nachher tritt wieder starke Hemmung ein, mit mehreren Ausfällen. $H$ vorüber, die Hemmung vorüber. Bew: das Tier bewegt sich, worauf (Hemmung) starke Hemmung mit einem Ausfall wieder eintritt. Bald nachher ist die Hemmung plötzlich vorbei. Zuckungshöhe dann konstant bis zum Ende der Kurve.

Kurve 19 (Taf.III.) (8. Oktober 1905). Esculenta. 48 Stunden vor dem Versuch und während desselben bei plus $27-28^{\circ} \mathrm{C}$. $N$ Nadel der Pravaz'schen Spritze durch die Hant eingeführt. Injektion $1 / 2 \mathrm{~cm} \mathrm{NaCl} \mathrm{10 \% .} \mathrm{Bew:} \mathrm{Bewegungen}$ des Tieres. .... $(Z)$ : neue Reihe anf dem Kymographion. $\ddot{o}$ (dreimal): Betupfung der Corneae. Das Tier reagiert auf die angegebenen Reize jedesmal mit stark vergrösserten Zuckungen; solche kommen auch zwischen den Reizungen vor.

6. Bei längeren Tetanis ationen $(20-30$ Sekunden) des gekreuzten Ischiadicus treten sowohl während der Reizung als auch im Augenblick nach dem Aufhören derselben bei Kältetieren einzelne Ausfälle wie auch längere Reihen von Ausfällen oder Frniedrigungen der Zuckungen ein. In der Regel lässt sich ein Zusammenhang zwischen der auf die Tetanisation eintretenden Hemmung und den Ausfällen von Zuckungen (resp. Erniedrigung derselben) konstatieren. Bei Wärmetieren kommt es, wie nach Kochsalzinjelktion, selten zu Ausfällen, in der Regel vielmehr wie dort zu erhöhten Zuckungen.

Kurve 20 a (Taf. III) (13. Dezember, 1905). Esculenta (Kaltfrosch). Anfang und Dauer der Tetanisation (R.-A. $=1 \mathrm{~cm}$ ) wird vom Signal angegeben. Während derselben starke Erniedrigung der Zuckungshöhen.

Kurve 20 b (Taf. III.) (ebenso). Tetanisation (R.-A. $=1 \mathrm{~cm}$ ) und Massage mehrere Sekunden schon vor dem Anfang des Kurvenabschnittes. Unterer Strich zeigt die Dauer der tetanischen Reizung, der obere die Dauer der Massage. Gleich nach dem Aufhören der Massage werden zwei Zuckungen erniedrigt; nach dem Aufhören der Tetanisation treten Ausfall und erhebliche Senkung der Zuckungshöhe ein.

b) Komplikation der Erregbarkeitsänderung des Muskelnerven mit tonischen $\mathrm{Phänomenen.}$

Mit den oben beschriebenen Verkleinerungen resp. Vergrösserungen der Zuckungshöhe können sich bei Kältetieren und normalen Fröschen (ausnahmsweise bei Wärmetieren) die oben beschriebenen tonischen Phänomene kombinieren. Es wurde ja oben hervorgehoben, dass die tonischen Kontraktionen resp. Verlängerungen 
bei rhythmischer Reizung des linken Ischiadicus in der Fusspunktslinie der Zuckungskurve zum Ausdruck kommen kann. Die tonischen Kontraktionen werden dabei meistens etwas höher und die Verlängerungen etwas deutlicher. Solche Kontraktionen treten besonders häufig bei Tetanisation des rechten Ischiadicus und bei Kochsalzinjektion mit Massage ein. Solange es sich um die tonischen Phänomene und die Veränderungen der Anspruchsfähigkeit des Nerven allein handelt, bereitet die Beschreibung keine Schwierigkeiten; sowie sich aber beide Phänomene miteinander kombinieren, treten wesentliche Komplikationen ein, weil man nicht berechtigt ist, ohne weiteres an eine algebraische Summation zu denken. Es scheint daher zweckmässig, die erhaltenen Resultate nach dem Gesichtspunkt des Steigens und Fallens der Gipfellinie und der Fusspunktslinie der Zuckungen $\mathrm{zu}$ beschreiben. Von Bedeutung ist bei diesen Kombinationen das Verhältnis, in welchem die Grösse der Veränderung der Gipfellinie und der Fusspunktslinie zueinander stehen. (Bei vollkommenem Ausfall von Zuckungen fallen Gipfellinie und Fusspunktslinie zusammen.)

Danach wären theoretisch möglich die folgenden Kombinationen:

1. Gleichbleiben der Gipfellinie; Erhöbung der Fusspunktslinie. Dieser Fall ist nicht sehr gewöhnlich und meistens tritt er nur bei ziemlich schwacher Reizung ein; bei Kochsalzinjektion habe ich ihn öfter beobachtet, aber auch bei Tetanisation des rechten Ischiadicus kommt er vor.

Kurve 21 (Taf. III.) (12. Dezember 1905). Esculenta (Kalttier). Das Signal gibt die Dauer der Tetanisation an. R.-A. des kleinen Induktoriums $3,5 \mathrm{~cm}$. Konstanz der Gipfellinie (relativ); Erhöhung der Fusspunktslinie.

Kurve 22 (Taf. III.) (7. Oktober 1905). Esculenta (Kalttier). Bei der ersten Marke wird die Nadel der Pravaz'schen Spritze durch die Rückenhaut geführt; bei der zweiten: Injektion $1 \mathrm{~cm} \mathrm{NaCl} \mathrm{10 \% .} \mathrm{Relative} \mathrm{Konstanz} \mathrm{der} \mathrm{Gipfellinie}$ (langsames Sinken der Zuckungshöhe von der Hemmung unabhängig); Erhöhung der Fusspunktslinie.

2. Gleichbleibender Gipfellinie; Senkungder Fussp unktslinie. Diese Kombination tritt meistens nur bei Wärmetieren in Erscheinung, und zwar habe ich sie nur bei faradischer Reizung des rechten Ischiadicus beobachtet. Die betreffende Senkung der Fusspunktslinie, die meistens auf die Dauer der Reizung beschränkt ist, tritt auch ein, ohne dass der Muskel während der Dauer des Versuches in tonische Kontraktion geraten war. Diese Erscheinung habe ich ohne rhythmische Reizung nicht beobachtet. In dieser 
meist geringen $(1 / 2-1 \mathrm{~mm})$ Senkung besteht in solchen Fällen gewöhnlich die einzige sichtbare Reaktion auf den zugeführten hemmenden Reiz, Es konnte daher auf Reproduktion einer solchen Kurve verzichtet werden.

3. Senkung der Gipfellinie; Erhöhung der Fusspunktslinie. Von allen bis jetzt beobachteten Fällen ist diese Kombination bei Kältetieren und normalen Tieren die gewöhnlichste. Sie wurde bei Tetanisation des gekreuzten Ischiadicus, bei Kochsalzinjektion in den Rückenlymphsack, bei Unruhe des Tieres und in anderen Fällen beobachtet. Besonders bei starker Hemmung, z. B. wenn die Wirkung einer Kochsalzinjektion durch Massage verstärkt wurde, ist diese Kombination die allergewöhnlichste (Kalttier).

Kurve 23 (Taf. III.) (13. Dezember 1905). Esculenta (Kalttier). Die Dauer der Faradisation wird in der Signallinie angegeben. Während der Reizung und unmittelbar nach derselben Senkung der Gipfellinie und Erhöhung der Fusspunktslinie.

Kurve 24 (Taf. III.) (9. April 1905). Esculenta. Kochsalzhemmung. $P$ : die Haut wird mit der Pinzette gefasst. $N$ : die Pravaz'sche Spritze eingefuhrt. Inj: Injektion von $0,4 \mathrm{ccm} \mathrm{NaCl} 10 \%$ in den Rückenlymphsack. Massage 1: Fusspunktserhöhung. Massage 2: gleich danach starke Erhöhung der Fusspunktsslinie, mehrere Ausfälle und Senkungen in der Höhe mehrerer Zuckungen. Langsamer Abfall der Fusspunktslinie bis auf das frühere Niveau. Bei Bev: Bewegungen des Tieres. Hemmungsdauer in der Kurre angegeben.

4. Senkung der Gipfellinie; Senkung der Fusspunktslinie. Diese Kombination habe ich sowohl bei Kältetieren wie Wärmetieren nach faradischer Reizung des gekreuzten Ischiadicus (ausnahmsweise bei Kochsalzinjektion) beobachtet. Das Phänomen ist bei Kältetieren am deutlichsten zu sehen.

Kurve 25 (Taf. IV.) (10. Dezember 1905). Esculenta (14 Tage bei plus $0-5^{0} \mathrm{C}$.). Das Tier reagiert sehr stark tonisch. Der linke Ischiadicus wird tetanisiert ( $0 \mathrm{~cm} \mathrm{R.-A.} \mathrm{des} \mathrm{kleinen} \mathrm{Induktoriums).} \mathrm{Dauer} \mathrm{der'Tetanisation} \mathrm{in} \mathrm{der} \mathrm{Signal-}$ linie angegeben. Mehrere Ausfälle und beträchtliche Senkung der Fusspunktslinie.

5. Erhöhung der Gipfellinie; Erhöhung der Fusspunkts linie. Diese Kombination wird sowohl bei Faradisation des Ischiadicus wie nach Kochsalzinjektion bei Kältetieren beobachtet.

Kurve 26 (Taf. IV.) (7. Dezember 1905). Esculenta (Kalttier). $M B$ (= meine Bewegung, s. oben S. 281) Tetanisation des linken Ischiadicus (R.-A. $0 \mathrm{~cm}$ des kleinen Induktoriums). Dauer der Reizung in der Signallinie angegeben. Starke Erhöhung der Fusspunktslinie und der Gipfellinie während der Reizung. 
In Kombination mit diesem Fall (5) beobachtet man nicht selten einzelne Ausfälle; besonders erscheinen diese oft, wenn wäbrend der hemmenden Reizung von anderen Körperteilen aus Erregungen ausgelöst werden.

Bei sehr stark tonisch-reagierenden Tieren tritt häufig eine Verschmelzung ein zwischen den vom Nerven ausgelösten Zuckungen und den reflektorischen Reizeffekten. Es kann so eine Art von Tetanus entstehen. Bei solchen Versuchstieren wirken aber nichtalle Reizfrequenzen bei der reflektorischen Reizung ebenso günstig für die Entstehung eines Tetanus. Es wurde mehrmals beobachtet, dass Faradisation des rechten Ischiadicus mit grosser Frequenz wirkungslos blieb; wenn aber der Schlüssel des primären Stromkreises so schnell wie möglich mit der Hand geöffnet und geschlossen wurde, trat Tetanus ein.

Kurve 27 (Taf. IV.) (14. Januar 1906). Temporaria (Kalttier). Bei $\alpha$ Tetanisation des gekreuzten Ischiadicus mit grosser Unterbrechungsfrequenz. Nachwirkung: eine sehr hohe Zuckung. Bei $w$ bedeutend kleinere Frequenz; Entstehung eines Tetanus.

6. Erhöhung der Gipfellinie; Senkung der Fusspunktslinie. Dieser Fall kommt am deutlichsten bei ziemlich stark tonisch reagierenden Tieren vor, und zwar bei Massage nach Kochsalzinjektion, und dann meistens in dem Stadium, wo die Hemmung nicht mehr sehr tief ist.

Kurve 28 (Taf. IV.) (14. Mai 1905). Temporaria. Vor dem Anfang der reproduzierten Kurvenabschnitte sind $0,4 \mathrm{ccm} \mathrm{NaCl} \mathrm{10 \%} \mathrm{mit} \mathrm{einer} \mathrm{Pravaz'schen}$ Spritze in den Rückenlymphsack eingeführt worden. Variationen in der Höhe der Gipfellinie, sowie ein langsames Ansteigen der Fusspunktslinie. Vom Anfang des Abschnittes bis 1 allmähliches Sinken der Gipfellinie und Erhöhung der Fusspunktslinie. Von 1-2 Massage der Rückenhaut; es tritt gleich Steigen der Gipfellinie und Sinken der Fusspnnktslinie ein. Nach Aufhören der Massage nähern sich Gipfellinie und Fusspunktslinie wieder. Von 3-4 Massage; sie hat dieselbe Wirkung wie zwischen 1 und 2. Nach Aufhören der Massage nähern sich Gipfellinie und Fusspunktslinie kaum mehr.

c) Weitere Komplikationen.

Die bisher beschriebenen Veränderungen der Zuckungskurve des vom Nerven aus gereizten Muskels auf die angegebenen hemmenden Reize hin können rein auftreten, d. h. das Bild bleibt während der ganzen Dauer der Reizung gleichartig. Es können aber auch während der Dauer der hemmenden Reize Veränderungen in dem Einfluss derselben auf die Zuckungskurve auftreten. 
Wenn die Veränderungen im Anfang der Kurve in einzelnen Ausfällen bestehen (normale und Kältetiere) resp. in einzelnen hohen Zuckungen im Rhythmus (bei Wärmetieren) und die Fusspunktslinie dabei keine Veränderung zeigt, so wird diese Veränderung nie von irgendeiner anderen im Verlaufe derselben gleich starken Reizung ersetzt. Ebenso verbält sich - mutatis mutandis - der Muskel meistens, wenn sich Ausfälle mit Senkung der Fusspunktslinie kombinieren.

Wenn aber die Fusspunktslinie in die Höhe geht oder gleich im Anfang nur etwas sinkt (ohne Veränderung der Gipfellinie), werden diese Anfangsbilder bald von anderen der oben angegebenen Kombinationen ersetzt.

A) Wird z. B. bei einem Kaltfrosch der rechte Ischiadicus tetanisiert und tritt eine tonische Kontraktion mit Konstanz oder Erhöhung der Gipfellinie auf, so kann diese tonische Kontraktion bis zum Ende der Reizung, wenn auch etwas schwächer wie im Anfang, bestehen bleiben; meistens geht jedoch diese tonische Kontraktion schnell zurück; es treten dann folgende Möglichkeiten auf:

1. Der Muskel kann sich ziemlich plötzlich schnell verlängern; die Kurve geht daher unter das Normalniveau ziemlich tief herunter und kehrt dann sehr langsam wieder auf das Normalniveau zurück. Zi der Zeit, wo die Fusspunktslinie unter dem Normalniveau steht, hat die rhythmische Reizung gar keine oder nur eine geringe Wirkung auf die Kurve. Allmählich aber wachsen die Zuckungen mehr und mehr und erreichen ihre frühere Höhe wieder.

Kurve 29 (Taf. IV.) (7. Dezember 1905). Esculenta (Kaltfrosch). Die Signallinie gibt Anfang und Dauer von 4 Faradisationen des rechten Ischiadicus an (R.-A. $=2 \mathrm{~cm}$ des kleinen Induktoriums). Bei 1 und 2 Entstehung einer starken tonischen Kontraktion, dann Senkung der Fusspunktslinie unter die Abszisse, Verkleinerung der Zuckungen, Zurückgehen auf die Abszisse (mehrere unbedentend höhere Znckungen im Rhythmus). Bei a Nachwirkung (siehe unten). 3 und 4 ebenso wie 1 und 2 ; die tonische Anfangskontraktion ist weniger ausgeprägt vorhanden.

2. Die Kurve geht nur auf das Normalniveau zurück und bleibt dort während der übrigen Reizung; die Zuckungen, die in dieser Lage der Fusspunktslinie auftreten, sind nicht selten erheblich reduziert, resp. können gänzlich wegfallen.

Kurve 30 (Taf.IV) (7. Dezember 1905). Esculenta (Kalttier). Anfang und Dauer der Faradisation des rechten Ischiadicus (R.-A. $=0 \mathrm{~cm}$ des kleinen Induktoriums) 
werden in der Signallinie angegeben. Tonische Kontraktion, Zurückgehen auf das Normalniveau mit einem Ausfall und Senkungen der Zuckungshöhe.

3. Die Kurve geht bald mehr oder weniger vollständig auf das Normalniveau zurück; sie bleibt dort während zwei oder drei Zuckungen, um dann wieder in einer tonischen Kontraktion in die Höhe zu gehen. Während der Zeit vom Anfang der ersten Kontraktion bis zum Ende der zweiten ist während dieser Kombination die Gipfellinie meistens etwas erhöht.

B) Antwortet das Tier unter denselben Versuchsbedingungen mit einer geringen Senkung der Fusspunktslinie, so kommt es, besonders bei stark abgekühlten Tieren, häufig vor, dass diese Kombination einer ziemlich plötzlichen gegenseitigen Näherung der Gipfellinie und der Fusspunktslinie Platz macht. Dieses Stadium der erhöhten Fusspunktslinie und gesenkten Gipfellinie dauert dann bis zum Ende der reflektorischen Reizung.

Kurve 31 (Taf. IV.) (7. Dezember 1905). Esculenta. Faradisation des rechten Ischiadicus $(0 \mathrm{~cm})$, Senkung der Fusspunktslinie, dann Erhöhung derselben. Kleine durchgängige Senkung der Gipfellinie. Bei a Nachwirkung (siehe unten).

C) Tritt nach einer Kochsalzinjektion als erste Reizantwort eine Erhöhung der Fusspunktslinie bei gleichzeitiger Senkung der Gipfellinie ein, so macht dieser Kombination später nicht selten einer Senkung der Fusspunktslinie und Erhöhung der Gipfellinie Platz.

Wenn ein Ischiadicus (rechts) eines Kältetieres, resp. eines normalen Frosches faradisch gereizt wird, so erfolgt fast immer eine tonische Kontraktion des gekreuzten (linken) Gastrocnemius. Wird dabei der linke Ischiadicus rhythmisch mit Einzelschlägen gereizt, so tritt für die Dauer der Reizung eine Erhöhung der Fusspunktslinie ein, und damit kombinieren sich meistens Variationen der Gipfellinie der Zuckungsreihe, wie oben gezeigt. Man beobachtet unter solchen Umständen nicht selten, dass die tonische Kontraktion resp. Erhöhung der Fusspunktslinie nicht sehr gross wird. Versucht man dann durch Erhöhung der Frequenz der Unterbrechungen oder durch Verkleinerung des Rollenabstandes die tonische Kontraktion resp. die Erhöhung der Fusspunktslinie zu vergrössern, so erweist sich auch die neue Stromintensität nicht wirksamer als die frühere: die tonische Kontraktion resp. die Erhöhung der Fusspunktslinie wird nicht grösser. Streichelt man dann aber sanft die Rückenhaut des 
Versuchstieres, so tritt sogleich eine sehr starke tonische Kontraktion resp. Frhöhung der Fusspunktslinie ein. (Man vergleiche die oben mitgeteilte Erfahrung, dass Versucbstiere, die sich in einer Kochsalzhemmung befinden, für Reize aus verschiedenen Körperteilen eine grössere Empfindlichkeit wie sonst besitzen.)

Kurve 32 (Taf. IV.) (7. Dezember 1905). Grosse Esculenta (Kalttier). 1. Obere Reihe. Bei $a$ hört die rhythmische Reizung des linken Ischiadicus auf; einige Sekunden nachher fängt die Faradisation des rechten Ischiadicus an (zuerst mit 2, dann mit einem und schliesslich mit $0 \mathrm{~cm} \mathrm{R.-A.} \mathrm{des} \mathrm{kleinen} \mathrm{Induktoriums);}$ im Anfang sinkt die Kurve etwas, von $b$ an steigt sie wieder langsam, von $M$ bis $c$ wird die Rückenhaut leicht massiert; die Kurve steigt sogleich in einer starken tonischen Kontraktion an. - 2. Untere Reihe. Bei $\gamma_{1}$ Anfang der Faradisation des rechten Ischiadicus (Rollenabstände $\gamma_{1} 2 \mathrm{~cm}, \gamma_{2} 1 \mathrm{~cm}, \gamma_{3} 0 \mathrm{~cm}$ ), $\delta$ bis $\varepsilon$ Massage: die Fusspunktslinie geht sogleich in die Höhe. Bei Empf. Nachwirkung (siehe unten).

\section{d) Die Nachwirkungen.}

Oben wurde bereits besprochen, dass nach dem Aufbören einer reflektorischen, faradischen Reizung eine einzelne Zuckung ausfallen kann. Sehr bäufig zeigen sich aber Nachwirkungen, welche sich auf längere Zeit erstrecken oder längere Zeit (40-80 Sekunden) nach dem Aufhören der Reizung eintreten.

Diese Nachwirkungen zeigen die oben besprochenen Formen, welche auch während der Reizung auftreten können; doch ist die nach der Reizung auftretende Wirkung oft eine andere als die, welche sich während der betr. Reizung zeigt. Solche Nachwirkungen kann man bei der Kochsalzhemmung nicht mit Sicherheit nachweisen, weil das Aufhören der Wirkung des Reizes nicht genau bestimmt werden kann. Anders bei der Faradisation des gekreuzten Ischiadicus.

Den Faradisationsversuchen sind folgende Angaben über die bis jetzt beobachteten Nachwirkungen entnommen worden:

\begin{tabular}{l|l}
\hline Effekt während der Reizung & Nachwirkung \\
\hline I. Konstanz der Fusspunktslinie: \\
1. mit hohen Zuckungen im \\
$\begin{array}{l}\text { Rhythmus der Reize; } \\
\text { 2. mit kleinen Variationen in der } \\
\text { Gipfellinie; }\end{array}$ & $\begin{array}{l}\text { Sohe Zuckungen in dem Rhythmus. } \\
\text { 3. mit Ausfällen resp. Gruppen Ausfällen. } \\
\text { von Ausfällen. }\end{array}$ \\
\end{tabular}




Effekt während der Reizung
II. Erhöhung der Fusspunktslinie:
1. mit Erhöhung der Gipfellinie;
2. mit Senkung der Gipfellinie;
3. nach her Senkung unter das
Normalniveau.
III. Senkung der Fusspunktslinie mit
Senkung der Gipfellinie.

Nachwirkung

Kurvenbeispiele.

Für die unter $\mathrm{I}(1,2,3)$ besprochenen Nachwirkungen dürfte es kaum nötig sein, Beispiele zu geben, da es ja aus anderen Kurvenabschnitten, die hier schon reproduziert sind, hervorgeht, wie die betreffenden Kombinationen aussehen.

II 1. Nachwirkung.
II 1. .

II 1.

II 2

II 3. a) neue Erhöhung der Fusspunktslinie (mit oder ohne Erhöhung der Gipfellinie);

b) einzelne Ausfälle;

c) hohe, zusammengeschmolzene Zuk-, kungen;

d) $\mathrm{b}+\mathrm{c}$;

Hohe, zusammengeschmolzene Zukkungen:

Hohe, zum Teil zusammengeschmolzene Zuckungen.

Einzelne Ausfälle und allgemeine Senkung der Gipfellinie.

Für die Nachwirkungen, die in der Tabelle mit III bezeichnet sind, dürfte aus demselben Grunde wie bei $\mathrm{I}(1,2,3)$ Reproduktionen nicht erforderlich sein.

Als Nachwirkungen sind auch die oben (S. 281) beschriebenen, in den Kurven $18 \mathrm{a}$ und $18 \mathrm{~b}$ wiedergegebenen Veränderungen in der Zuckungshöhe nach Unruhe des Versuchstieres zu betrachten:

Diese Nachwirkungen treten etwa in der Hälfte der Fälle direkt nach dem Aufhören des Reizes ein. In anderen Fällen etablieren sie sich erst etwa $40-80$ Sekunden nach demselben; im letzteren Falle ist das Stadium der Nachwirkungen in der Regel kurz.

Im ersteren Falle, besonders bei I, 3, dauert das Stadium der Nachwirkung meist sehr lange und kann sogar während der ganzen 
Dauer des Versuchs anhalten; d. h. die Schwelle des Nerven hat sich infolge der einmaligen hemmenden Rejzung andauernd in negativem Sinne verändert, und man muss, um wieder konstante Zuckungen zu bekommen, den Rollenabstand verkleinern, manchmal um $2 \mathrm{~cm}$.

\section{Literatur.}

Inwiefern die Erregbarkeit der vorderen Rückenmarkswurzeln von dem Erregungszustand der hinteren abhängig ist, dürfte meines Wissens zum erstenmal von $\mathrm{Harless}^{1}$ ) als Problem aufgestellt worden sein. Er fand, dass die Erregbarkeit des motorisch-sensorischen Nervenstammes nach der Durchschneidung der sensiblen Wurzeln steigt. Harless konstatierte auch, dass die Erregbarkeit, welche nach Durchschneidung der sensiblen Wurzeln gestiegen war, sank, wenn er die zentralen Wurzelstücke mit Kochsalzlösung reizte.

Zu einem positiven Resultat kam auch 1865 E. von Cyon ${ }^{2}$ ) (die Untersuchungen von Harless werden nicht erwähnt). Die einzige Verschiedenheit der Methodik zwischen Harless und v. Cyon besteht darin, dass, während Harless den gemischten Nervenstamm mit Schliessungs- und Öffnungsschlägen eines durch einen Rheostat abgestuften konstanten Stromes reizte, v. C y o n die vorderen Wurzeln mit eben wirksamen Induktionsschlägen erregte. v. Cy on kam zu dem Resultat, dass die Durchschneidung der hinteren Wurzeln einen senkenden Einfluss auf die Erregbarkeit hervorruft. Im Anschluss an diesen Befund erörterte er die Frage, $o b$ das Phänomen dureh den so umstrittenen Brondgeest'schen reflektorischen Tonus erklärt werden könnte. Er ging dabei gewiss von der Vorstellung aus, dass ein Nerv, der sich schon in schwachem Erregungszustand befindet, um eine Kontraktion im zugehörigen Muskel anszulösen, einen kleineren Reizzuwachs nötig hat als ein Nerv, der sich gar nicht im Erregungszustande befindet. Da die reflektorische Erregbarkeit nach Entfernung der höheren Zentren steigt, hatte v. Cy on erwartet, dass eine Erböhung der Erregbarkeit der vorderen Wurzeln durch die gewöhnliche quere Trennung des Rückenmarkes vom Gehirn zustande kommen würde; die Versuche ergaben aber

1) Harless, 1. c. III (Abhandl. d. math.-phys. Klasse bayer. Akad. d. Wiss. Bd. 8 S. 683). 1860 .

2) E. v. Cy on, Über den Einfluss der hinteren Nervenwurzeln des Rückenmarks auf die Erregbarkeit der vorderen. Ber. über d. Verh. d. königl. sächs. Gesellsch. d. Wiss. zu Leipzig. Math.-phys. Klasse. Bd. 17 S. 82-95. 1865. 
gerade das entgegengesetzte Resultat: die Erregbarkeit sank. Harless, der dies auch gesehen batte, gibt an, dass die Senkung durch eine lang andauernde Steigerung abgelöst wird ; davon hatte v. C y on aber nie etwas gesehen. v. Cy on hatte auch gefunden, dass, wenn die hinteren Wurzeln durchschnitten waren, die Entfernung der höheren Zentren ohne Einfluss war.

A. v. Bezold und Uspensky ${ }^{1}$ ) bestritten die Richtigkeit der Beobachtungen von v. Cyon. Die genannten Forscher fanden, dass die an und für sich nach Freilegung des Rückenmarkes eintretende Senkung der Erregbarkeit der vorderen Wurzeln nicht wesentlich schneller wurde nach Durchschneidung der sensiblen Wurzeln der anderen Seite, sondern ganz im Gegenteil wurde meistens eine Verlangsamung der Senkung nach der Durchschneidung beobachtet. Nur in ein paar Fällen wäre der Verlauf den Angaben v. Cy on's entsprechend gewesen; das sollte dann von Fehlerquellen herrühren. Dagegen konnten v. Bezold und Uspensky eine schwache Erregbarkeitserhöhung durch Reizung der hinteren Wurzeln derselben Seite hervorrufen. Dies Resultat bekamen sie bei Hautreizung trotz einer durch Curarezufuhr bedingten Lähmung resp. gänzlichen Ruhe des Tieres. Das reagierende Glied war durch Unterbindung der Gefässe vor Curarevergiftung geschützt. Die Verfasser leugnen auf Grund dieser Versuche jede "besondere" Einwirkung der hinteren Wurzeln auf die vorderen.

v. $\mathrm{Cy} \mathrm{on}{ }^{2}$ ) lenkte dann die Aufmerksamkeit darauf, dass bei dieser eben referierten Untersuchung v. Bezold und Uspensky sich ihre Vorstellungen aus Versuchen an curarisierten Fröschen gebildet hätten; erst nachträglich hätten sie ihre Auffassung auch bei nicht vergifteten Tieren bestätigt gefunden. - Kurz nachher konnte auch $G u t \operatorname{man} n^{3}$ ) nach eigenen Untersuchungen die Angaben von v. Cy on bestätigen. Bei dem langsamen Sinken der Erregbarkeit der vorderen Wurzeln nach Freilegen des Rückenmarkes fand er

1) A. v. Bezold und Uspensky, Über den Einfluss der hinteren Rückenmarkswurzeln auf die Erregbarkeit der vorderen. Zentralbl. f. d. med. Wiss. Bd. 5 S. $611-613,819-821.1867$.

2) E. v. Cyon, Über den Einfluss der hinteren Wurzeln auf die Erregbarkeit der vorderen. Zentralbl. f. d. med. Wiss. 1867 S. $643-645$.

3) P. Gutmann, Zur Lehre von dem Einfluss der hinteren Rückenmarkswurzeln auf die Erregbarkeit der vorderen. Zentralbl. f. d. med. Wiss. 1867 S. $689-690$. 
nach der Durchschneidung der sensiblen Wurzeln der anderen Seite ein entschieden viel schnelleres, plötzlicheres Sinken der Erregbarkeit. - Auch F. Stein man ${ }^{1}$ ), der unter der Leitung von v. Cy on arbeitete, konnte bald nachher die Beobachtungen seines Lehrers bestätigen.

Gegen die Ausführungen von Harless machte dann Grünhagen ${ }^{2}$ ) geltend, dass Harless den gemisehten Nerven gereizt hätte, also auch die sensiblen Fasern. Auch wird betont, dass eine Beeinflussung der Erregbarkeit der vorderen Wurzeln durch die hinteren Wurzeln nicht mit seiner Theorie für die Summation zweier Reize stimmt.

Das Problem war nun durch die Reflexionen v. Cy on's eine Teilfrage geworden im Kampf um den Brondgeest'schen Tonus. Schon früher hatten ja Heidenhain ${ }^{3}$ ) und Auerbach ${ }^{3}$ ) am Reflexpräparat zeigen können, dass die Durchschneidung und das Leitungsunfähigmachen des Nerven in keiner Weise die Länge des Muskels beeinflusste; $\mathrm{Schwalbe} \mathrm{e}^{3}$ ) hat diese Beobachtung mit den feinsten Methoden bestätigt. Der so entstandene Skeptizismus gegen alles was Tonus heisst, erstreckte sich auch auf die Frage, die uns hier beschäftigt. Unter Grünhagen's Leitung kontrollierte G. Heidenhain ${ }^{4}$ ) die Angaben von v. Cyon, Gutmann und Steinmann. Es wird hervorgehoben, dass die Verschiebung der Elektroden auf den Nerven eine Hauptfehlerquelle bei den genannten Untersuchungen gewesen wäre - was v. $\mathrm{Cy} \circ \mathrm{n}^{5}$ ) in einer Antwort zurückweist. Unter Anwendung von verschiedenen Kautelen kommt Heidenhain dann zu dem Resultate, dass nach der Freilegung des Rückenmarkes keine Senkung der Erregbarkeit eintritt, und dass die Durchschneidung der hinteren Wurzeln keinen Einfluss auf die Erregbarkeit der vorderen hat. Stein $\operatorname{man}^{\mathbf{1}}$ ) hat in seiner oben schon erwähnten Arbeit auch einen Versuch mitgeteilt, der folgendermassen ausgeführt wurde: Bei einem am Unterkiefer auf-

1) F. Ste inmann, Üher den Tonus der willkürlichen Muskeln. Mélanges biologiques tirés du Bull. de l'Acad. etc. de St. Pétersbourg t. 7 p. 787.1871 zit.) nach Eckhard).

2) Grünhagen, Bemerkungen über die Summation von Erregungen in der Nervenfaser. (Zeitschr. f. ration. Medic. 3te R. Bd. 26. 1866. S. 190-224).

3) 1. c. (S. 29).

4) G. Heidenhain, Über den Einfluss der hinteren Rückenmarkswurzeln auf die Erregbarkeit der vorderen. Pflüger's Arch. Bd. 4 S. 435-453. 1871.

5) E. v. Cyon, Über den Einfluss usw. Pflüger's Arch. Bd. 8 S. 347 bis 348.1874. 
gehängten normalen Tier ist die Sehne eines Gastrocnemius, z. B. links, freigelegt und der Muskel durch ein daran befestigtes Gewicht belastet; werden nun die hinteren Wurzeln durchschnitten, so sinkt das Gewicht gleich. Diesen indirekten Beweis macht Heidenhain nicht nach, da doch die ganze Tonusfrage nach der Arbeit von Schwalbe erledigt wäre.

Nach dieser Arbeit blieb die Frage lange liegen; Marcacei ${ }^{1}$ ) nahm sie 1882 auf und konstatierte die Richtigkeit der Harlessschen Angaben. 1883 veröffentlichte $\mathrm{K}$ anelli s${ }^{2}$ ) eine kurze Mitteilung über denselben Gegenstand und mit demselben Resultat wie Harless und Marcacei.

Erst 1890 wurde das Problem wieder in Angriff genommen, und zwar von italienischen Forschern. Hier dienten Sängetiere als Versuchsobjekte.

Belmondo und $\mathrm{Oddi}^{3}$ ) legten bei den Versuchstieren die Rückenmarkswurzeln frei; die motorischen Wurzeln wurden auf Elektroden gelegt, und die Muskulatur des zugehörigen Beines war durch einen "tambour de Marey" mit einer Schreibvorrichtung verbunden und diente als Index für die Erregbarkeit des Nerven. Die hinteren Wurzeln wurden mit Kokain-Chlorhydrat (10\% iger Lösung) bepinselt, um ihre Leistungsfähigkeit aufzuheben. Es trat dabei sehr schnell eine Verminderung der Anspruchsfähigkeit des Nerven ein. [Bei der Applikation des Kokains auf den Nerven tritt eine tonische Kontraktion in der Kurve in Erscheinung (siehe die Kurven der Autoren!). Dieses Pbänomen wird von den Verfassern nicht beschrieben.] Wurden bei einem Tier, dessen hintere Wurzeln kokainisiert waren, dieselben durchschnitten, so trat eine nochmalige Senkung der Erregbarkeit der vorderen ein. Wurden die Wurzeln durchschnitten, ohne vorher kokainisiert worden zu sein, so trat in gewissen Fällen eine starke Erhöhung ein, die aber bald einem langsamen Sinken Platz machte. In anderen Fällen trat zuerst eine Senkung ein, wonach die Erregbarkeit wieder höher wurde; sie blieb jedoch konstant kleiner als vor der Durchschneidung. Diese beiden Erscheinungen konnten sich so kombinieren, dass zuerst grosse Er-

1) Marcacci, Nuovo fatto in favore della sinergia funzionale delle paia spinali. Archivio per le scienze med. vol. 5 No. 16. 1882 (zit. nach Polimanti).

2) Kanellis, Influences des racines sensitives sur. l'excitabilité des racines motrices. Compt. Rend. de la Acad. des Sciences. 23 avril 1883.

3) Belmondo e Oddi, 1. c. (S. 249). 
höhung, dann grosse Senkung eintrat, worauf die Erregbarkeit wieder in die Höhe ging, blieb aber konstant kleiner, wie vor der Durchschneidung der sensiblen Wurzeln. In noch anderen Fällen trat zuerst eine kleine Senkung ein, die einer lang andauernden Erhöhung Platz machte. Wurden während dieser langen Erhöhung der Erregbarkeit die zentralen Stücke der sensiblen Wurzeln kokainisiert, so verschwand wieder die Erregbarkeitserhöhung. Direkte Reizung der hinteren Wurzeln ergab eine ziemlich grosse Erhöhung der Anspruchsfähigkeit des peripheren Nerven. Die Verfasser konnten diese Variationen im Erfolg zum Teil auf technische Verschiedenheiten zurückführen; einfache Durchschneidung der hinteren Wurzeln mit einem scharfen Instrument sowie die Kokainisierung derselben rief jedesmal eine Senkung hervor; eine Erhöhung kam dagegen regelmässig zustande nach einer quetschenden Durchtrennung derselben. So wäre nach der Meinung der Autoren auch der Unterschied im Resultat zwischen v. Cyon und Harless zu erklären.

Manelli $i^{1}$ ) vermied bei seinen Versuchstieren (Katzen) die Blosslegung des Rückenmarkes und reizte den einen Ischiadicus, resp. einen Ast aus dem Brachialplexus der entgegengesetzten Seite mit faradischen Strömen, resp. einer Ligatur. Als Mass der Erregbarkeit des anderen Ischiadicus dienten die Kontraktionen in der Beinmuskulatur, die durch rhythmische Reizung vom Nerven aus ausgelöst wurden. Der Verfasser kam zu dem Resultate, dass durch die Reizung des einen Ischiadicus in gewissen Fällen eine Senkung der Erregbarkeit im anderen hervorgerufen werden kann (Zuckungshöhe sowie Tonus wurden verkleinert). In anderen Fällen wirkten die Reizungen „dynamogen" auf die Effekte der rhythmischen Reizung und auf den Tonus; beide wurden vergrössert. Die depressive sowie die fördernde Wirkung trat nicht gleich nach dem Anfang der Reizung ein und dauerte auch mehrere Sekunden länger als die Faradisation. Die Erregbarkeitssenkung konnte bis zu einigen Zentimetern Rollenabstand betragen.

Polimanti ${ }^{2}$ ) kam 1896 durchgängig zu dem Resultate, dass Reizung des einen Ischiadicus (beim Hund) unter allen Umständen einen hemmenden Einfluss auf die Erregbarkeit des anderen ausübt.

1) Manelli, l. c. (S. 250).

2) O. Polimanti, Influenza che le radici posteriori esercitano sulla eccitabilità delle anteriori. Bull. Acad. med. di Roma t. 221896 (Sep.). 
Durch Reizungen von der Hirmrinde aus "kónnten odd i ${ }^{1}$ ) (1895 und 1898) und Tomasini2) (1894) zeigen, dass die Erregbarkeit des peripheren Nerven vom Zentralnervensystem aus sich beeinflussen lässt.

Ähnliche Resultate teilte L. M. Patrizis) in bezug auf die Innervation des Zwerchfells mit. Er gab u. a. an, dass bei elektrischer Reizung der zentralen Vagusenden (beim Hund) die Wirkung künstlicher Reizung des N. phrenicus (rhythmische Einzelinduktionsschläge) vermindert wird. Die Zwerchfellbewegungen wurden mit einem "Phrenographen" registriert.

In seinem Sammelreferat über "Die intrazentralen Hemmungsvorgänge in ihrer Beziehung zur Skelettmuskulatur" (1902) referierte H. E. Hering ${ }^{4}$ ) über Kontrollexperimente, die er den Angaben Patrizi's entgegenstellt. Er registrierte dabei die Atembewegungen des Versuchstieres (in üblicher Weise) und benutzte statt der Vagusreizung den Trigeminusreflex, welcher durch Einblasen von Rauch in die Nase des unvergifteten Versuchstieres (Kaninchen) ausgelöst wurde. Die Phrenici wurden nicht rhythmisch gereizt, sondern die Wirkung des Minimalreizes vor und während des Stillstandes verglichen. "Das Versuchsergebnis schien auf den ersten Blick allerdings dafür zu sprechen, dass während des reflektorischen Atemstillstandes die Phrenici weniger anspruchsfähig, bezw. nicht anspruchsfähig seien. Ich" erkannte aber sogleich," sagt der Verfasser (S. 524), „dass dieses Resultat vo rg etä u scht wurde durch die bei Auslösung des Reflexes gleichzeitig erregten Exspiratoren, welche mechanisch das Merkbarwerden der nach wie vor vorhandenen, durch Induktionsschläge ausgelösten Zwerchfellzuckungen an der Atemkurve verhinderten bezw. dieselben kleiner

1) R. Oddi, Il cervello ed il midollo spinale come centri di inibizione. Rend. della R. Acad. dei Lincei t. 4 serie $5^{a}$, fasc. $6^{\circ}$ (Sep.). $-L^{\prime}$ inibizione dal punto di vista fisio-patologico, psicologico e sociale. Torino 1898.

2) S. Tomasini, L' eccitabilità della zona motrice dopo la recisione delle radici spinali posteriori. Atti dell' XI. Congr. Medico Intern. 1894. Sezione Fisiologica t. 2 p. $68 \mathrm{f}$.

3) L. M. Patrizi, Sur l'addition et l'élision entre les incitations naturelles et les incitations artificielles, dans les mourements du diaphragme; Archives italiennes de la biol. vol. 241896 p. 1.

4) H. E. Hering, Die intrazentralen Hemmungsvorgänge in ihrer Beziehung zur Skelettmuskulatur. Sammelreferat in den Ergebnissen der Physiologie, 1. Jahrg. Bd. Biophysik (S. 503-533) $523 \mathrm{f}$.

E. Pfiüger, Archiv für Physiologie. Bd. 114. 
erscheinen liessen, als sie in -der Tat waren." Der Verfasser überzeugte sich davon durch Ausschaltung der Bauchmuskulatur; unter diesen Umständen war die Reizung während des Stillstandes ebenso wirksam wie vorher.

Kürzlich erschien aus dem Institute Patrizi's eine Nota preventiva von $\operatorname{Franchini}^{1}$ ), in der diese Kritik zurückgewiesen wird. Der Verfasser zeichnete unter weitgehender Eröffnung der Bauchdecken mit dem „Phrenographen“ nur die aktiven Diafragmakontraktionen auf und zeigt in mehreren Kurven den erregbarkeitssenkenden Einfluss der Vagusreizung. Da H er ing die phrenographische Methode von Patrizi nicht benutzt hat, wird man seinen Einwand als widerlegt betrachten dürfen.

1898 veröffentlichte dann $\mathrm{Starke}^{2}$ ) die Publikation, über die ich schon oben in der Einleitung referiert habe. Auf die Verwornsche $^{3}$ ) Kontrolluntersuchung der Angaben Starke's, die in ihrem Resultat ganz und gar negativ war, muss ich hier etwas näher eingehen. Das Hauptsächliche der Resultate und der Methode ist schon aus der Einleitung bekannt. Ich will auf einige Punkte hindeuten, die wahrscheinlich - vom Standpunkt eines, der nicht dabei war, wahrscheinlich - die Ursache seines negativen Resultates gewesen sind.

Erstens, da V erw or n den Nerv mit eben wirksamen Öffnungsinduktionsschlägen reizt, so hat er gewiss die Stärke so gross genommen, dass die Variationen im Reizerfolg, welche ich oben Schwellenschwankung nannte, nicht mehr zu sehen waren. Aber diese anfänglichen Variationen sind eben, wie ich oben gezeigt habe, zu einem grossen Teil vom Zentralnervensystem abhängig. Auf diese Weise kam Verworn zu einer Schlagstärke, bei der er die betr. Phänomene kaum hat sehen können.

Zweitens scheint mir Verworn viel zu wenig die kolossale Hemmung durch die Fesselung berücksichtigt zu haben (Schrader's Hypnose). Er führt als Beweis dafür, dass seine Versuchstiere nicht gehemmt waren, die Tatsache an, dass sie beim Kneifen des einen Beines das audere angezogen haben. Nun leugnet wohl niemand,

1) G. Franchin i, L' eccitabilità frenico-diaframmatica durante la sospensione respiratoria di Franke. Comm. alla Sociétà dei Naturalisti e Matematici di Modena. Serie IV vol. 8 il 13 Febbraio 1906.

2) J. starke, 1. c. (S. 2).

3) M. Verworn, Zur Physiologie der nervösen Hemmungserscheinungen. Arch. f. Physiol. Supplem. 1900 S. 105-124. 
dass ein sog. hypnotisierter Frosch sich in tiefer Hemmung befindet, und doch dreht er sich schon, wenn er nur leicht angeblasen wird, wieder um und läuft seines Weges. Bei gefesselten Tieren kommt das Zentralnervensystem überhaupt nicht aus der Hemmung heraus, da der Widerstand bei den Bewegungen gleich eine neue Hemmung setzt. Wenn man dann die Tatsache dazu nimmt, die ich oben angegeben habe, dass ein (normales) Tier, das in unbequemer Stellung gefesselt ist, oft nur mit einer leichten Tonussenkung und sonst nicht reagiert, so dürften die negativen Resultate Verworn's wenig befremdend wirken.

\section{Theoretisches. Zusammenfassung.}

Ich habe oben gezeigt, dass auf reflektoriscbe Reize hin zwei verschiedene Erfolge am Muskel eintreten können: reflektorische schnelle Zuckungen und lang andauernde tonische Kontraktionen (Regel der doppelten Möglichkeit) [S. 256]. Hieraus ergibt sich die Wahrscheinlichkeit, dass der tonische Zustand des Muskels auf Erregung von seiten des Zentralorgans zurückzuführen ist. Hierfür ist auch anzuführen, dass die tonische Kontraktion absinkt, wenn die Leitung im zuführenden Nerven durch Anelektrotonus aufgehoben wird und nach Aufhebung desselben wieder eintritt [S. 261]. Bindend für die Annahme einer Dauererregung von seiten des Zentralorgans ist dieser Versuch nicht, denn es könnte sich beim Tonus sehr wohl im Sinne v. Uexküll's ${ }^{1}$ ) um eine einmalige Veränderung im Nerven handeln, die aber durch den Anelektrotonus rückgängig gemacht wird. Immerhin ist es wohl im Augenblick noch wahrscheinlicher, dass beim Tonus eine Dauererregung vom Zentrum aus dem Muskel zufliesst.

Es liegen nach Verwor $\mathrm{n}^{2}$ ) für das Zustandekommen nervöser Hemmung folgende drei Möglichkeiten vor:

1. Die motorische Erregung der Vorderhornzellen, welche die Kontraktion erzeugt, wird selbst gehemmt, und infolge des Weg-

1) J. v. Uexküll, Studien über den Tonus. I. Zeitschr. f. Biol. Bd. 44 (Sipunculus). - Studien über den Tonus. II. Zeitschr. f. Biol. Bd. 46 (Schlangenstern). - Studien über den Tonus. III. Zeitschr. f. Biol. Bd. 46 (Blutegel). Leitfaden in das Studium der experimentellen Biologie der Wassertiere. Wiesbaden 1905 . Vor allem S. 32-72.

2) M. Verworn, Zur Physiologie der nervösen Hemmungserscheinungen. Arch. f. Physiologie. Supplem. S. 105-124. 1900. 
falls oder Ausbleibens ihrer motorischen Impulse hört die kontraktorische Erregung des Muskels von selbst auf bezw. bleibt einfach aus.

2. Ausser den motorischen "Neuronen" versorgen den Muskel noch besondere "Hemmungsneurone", welche durch ihre Impulse den kontrahierten Muskel hemmen, d. h. eine bestehende Kontraktion unterdrücken bezw. den Eintritt einer Kontraktion verhindern.

3. Die motorischen Vorderhornzellen besorgen selbst die Hemmung des Muskels aktiv, indem sie bei ihrer ejgenen Hemmung einen aktiven Hemmungsvorgang, der dem motorischen Impulse qualitativ entgegengesetzt ist, dem Muskel durch ihre Achselzylinder übermitteln.

Um zwischen 1. einerseits und 2. und 3. anderseits eine Entscheidung treffen zu können, hat Verworn die Erregbarkeit des peripheren Nerven während der Hemmung untersucht. Wenn keine Veränderung in der Anspruchsfähigkeit des Nerven eintritt, so muss die Hemmung rein intrazentral sein. Für die letztgenannte Eventualität sprachen die Versuchsergebnisse Verworn's.

Die Resultate v. Cyon's und der italienischen Forscher sowie die hier oben referierten Versuchsergebnisse zeigen aber mit aller wünschenswerten Klarheit, dass eine Veränderung der Anspruchsfähigkeit während der Hemmung da ist. Es brauchen aber darum noch nicht die Eventualitäten 2. und 3. richtig zu sein. Beth ${ }^{1}$ ) hat schon darauf hingewiesen, dass eine Beeinflussung der Erregbarkeit so zustande kommen könnte, dass die grosse Menge "kleiner Reize", die sich zum Teil als Tonus der Muskulatur, zum Teil bei Zufuhr direkter Nervenreize in einer erhöhten Erregbarkeit geltend machen, also dynamogen wirken, im Zentrum durch den hemmenden Reiz abgeschnitten würde; eine Deutung, die zuerst von v. C y o n ausgeführt wurde und die die italienischen Forscher anzunehmen am meisten geneigt erscheinen.

Genügt diese Möglichkeit? Wenn die in den Kurven bei hemmenden Reizen auftretenden Veränderungen nur in einer Herabsetzung der Anspruchsfähigkeit und eventuell nebenher in einem Sinken des Tonus besteben, so kann man mit dieser Erklärung vollkommen auskommen. Derartige Veränderungen sind von v. Cyon, den italienischen Autoren und von mir (Kurven 25, 29) gefunden worden. Es kommen aber auch (mit Ausnahme von Warmfröschen) sehr häufig Kombinationen von Verminderung der Anspruchsfähig-

1) A. Bethe, 1. c. S. 382 f. 
keit und Erhöhung des Tonus vor (z. B. Kurven 23, 24). Wenn nun, wie wahrscheinlich ist, der Tonus in einem dem Muskel durch den Nerven dauernd zugeleiteten Erregungsprozess besteht, s o kann hier die Verminderung der Anspruchsfähigkeit des motorischen Nerven für direkte Reizung nicht allein daraufberuhen, dass derhemmende Reiz die schwachen dy namogenen Reize intrazentral abschneidet. Das häufige Vorkommen einer intrazentralen Hemmung wird damit selbstverständlich nicht geleugnet.

Um das betreffende Phänomen deuten zu können, muss man entweder annehmen, dass es hemmende Fasern gibt, die die Anspruchsfähigkeit herabsetzen, dabei aber die Fähigkeit der motorischen Fasern, tonische Reize zu leiten, nicht unbedingt aufheben; oder im motorischen Nerven werden verschiedenartige Prozesse geleitet, und die tonische und die schnelle Erregung beruhen auf ungleichartigen Nervenprozessen.

Durch die hier beschriebenen Phänomene ist meines Erachtens eine Brücke geschlagen zwischen den Wirkungen der zentralen Hemmung (Hemmung vom Zentralnervensystem aus innervierter Muskeln durch rezeptorische Reize) und den Hemmungswirkungen, welche bestimmte Nerven auf selbständige periphere Organe ausüben, besonders zu den Wirkungen des Vagus auf das Herz. Ich will in diesem Zusammenhang nur ganz kurz darauf hinweisen, dass sich das Herz in verschiedenen Beziehungen zu dem Vagus resp. Accelerans so verhält, wie ein Gastrocnemius mit dem gleichseitigen Nerven und Zentrum zu dem Ischiadicus der anderen Seite. Es geht ja aus dem Obigen hervor, dass positiv und negativ inotrope Wirkungen in der Zuckungskurve zum Vorschein kommen, und zwar kann manchmal die negativ jnotrope Wirkung so stark sein, dass der Muskel, trotz rhythmischer Reizung seines Nerven, eine kurze Zeit in Ruhe verharrt. Vom Isehiadicus resp. Vagus können ton is $\mathrm{ch}$ e Veränderungen in der Muskulatur des Gastrocnemius resp. Herzens ( $\mathrm{Fano}$ ) hervorgerufen werden. Beim $Z$ entralnervensystem ist eine refraktäre Phase ( $Z$ wa ardemaker, Bethe, Sherrington, Uexküll u. a.) nachgewiesen; kompensatorische Pause ist von mir (bei noch nicht veröffentlichten Versuchen) beim Atmungszentrum (des Aales) gesehen worden. Das Alles-oder Nichts- 
Gesetz ist (nach Biedermann) für das Rückenmark erheblich abgekühlter Tiere gültig gefunden. In einer späteren Arbeit werde ich die Analogie zwischen Vagus + Herz einerseits und sensible Nerven + Zentralnervensystem und Skelettmuskel anderseits weiter durchführen und so noch ein Argument zu den vielen fügen, die die Existenz eines nervösen Zentrums zwischen Herznerven und Herzmuskulatur wahrscheinlich machen.

\section{Zusammenfassung.}

Die hier referierten Untersuchungen ergeben folgendes:

1. Falls dem Zentralnervensystem eine Erregung zugeführt wird und dabei ein Effekt eintritt, erfolgt entweder eine schnelle Reflexzuckung von grosser Hubhöhe oder eine lang ausgezogene tonische Kontraktion mit geringer Hubhöhe. Diese tonischen Kontraktionen können durch Haut- und Nervenreize sowie auch dureh photische Reize hervorgerufen und, wenn sie eingetreten sind, durch andere Reize oder auch durch Wiederholung des gleichen wieder aufgehoben werden. Sie sind bei abgekühlten Tieren grösser und häufiger als unter normalen Umständen.

Der tonische Kontraktionszustand im Muskel wird durch Anelektrotonus in seinem Nerven aufgehoben und kehrt nach Strom. öffnung wieder zurück. Wenn nach starker Dehnung der Muskel eine Verlängerung zeigt, so kann er auf reflektorische Reize hin seine Normallänge ohne Zuckung (im Gegensatz zum Nervmuskelpräparat) wieder erlangen.

2. Auf reflektorische, hemmende Reize hin kann die Anspruchsfähigkeit des peripheren Nerven, und zwar sowohl in positivem wie in negativem Sinne, beeinflusst werden; gewöhnlich findet das letztere statt. Die Wirkung nicht zu kurz dauernder hemmender Reize kann in Kobinationen bestehen zwischen tonischen Veränderungen und Variationen in der Anspruchsfähigkeit des Muskelnerven; in vielen Fällen, besonders bei Wärmetieren, treten aber tonische Veränderungen nicht auf. Jeder starke, hemmende Reiz ruft ausser dem Effekt während der Reizung unmittelbar nach dem Aufhören der Reizung oder nach einem kurzen Intervalle eine Nachwirkung hervor.

3. Die beschriebenen Phänomene zeigen, dass nicht alle Hemmungen rein intrazentral verlaufen. $\mathbf{Z u}$ deren Erklärung sind entweder verschiedenartige Nervenprozesse oder ein Unterschied zwischen hemmenden und erregenden Fasern in den Muskelnerven anzunebmen. 